\title{
Nonparametric Significance Testing
}

\author{
Pascal Lavergne* \\ INRA-ESR
}

\author{
Quang Vuong \\ University of Southern California and INRA-ESR
}

\author{
December 95 \\ Revised June 1998
}

Financial support from INRA and the National Science Foundation under Grant SBR-9631212 is gratefully acknowledged. Earlier versions were presented at Université Toulouse 1, CentERTilburg, University Carlos III-Madrid, CREST-Paris, INRA-Jouy-en-Josas, ESEM 96-Istanbul, Séminaire Paris-Berlin 96, UC San Diego, University of Southern California and Texas Camp Econometrics. We thank participants, three referees and the co-editor for helpful comments.

*Corresponding address: INRA-ESR, BP 27, 31326 CASTANET-TOLOSAN Cedex FRANCE. E-mail address: lavergne@toulouse.inra.fr 


\begin{abstract}
A procedure for testing the significance of a subset of explanatory variables in a nonparametric regression is proposed. Our test statistic uses the kernel method. Under the null hypothesis of no effect of the variables under test, we show that our test statistic has a $n h^{p_{2} / 2}$ standard normal limiting distribution, where $p_{2}$ is the dimension of the complete set of regressors. Our test is one-sided, consistent against all alternatives and detect local alternatives approaching the null at rate slower than $n^{-1 / 2} h^{-p_{2} / 4}$. Our Monte-Carlo experiments indicate that it outperforms the test proposed by Fan and Li (1996).
\end{abstract}

Keywords: Hypothesis testing, Kernel estimation, Nested models.

JEL classification: Primary C52; Secondary C14.

\title{
Résumé
}

Une procédure pour tester la significativité d'un sous-ensemble de régresseurs dans un modèle de régression non-paramétrique est proposée. Elle s'appuie sur la méthode du noyau. Sous l'hypothèse nulle, i.e. lorsque les variables considérées ne sont pas pertinentes, la statistique de test a une distribution asymptotique normale en $n h^{p_{2} / 2}$, où $p_{2}$ est le nombre total de régresseurs. Le test est unilatéral, convergent contre toute alternative et détecte des alternatives locales qui s'approchent de $1^{\prime}$ 'hypothèse nulle à une vitesse inférieure à $n^{-1 / 2} h^{p_{2} / 4}$. Pour des petits échantillons, notre test a de meilleures performances que celui proposé par Fan et Li (1996).

Mots-Clés: Test d’hypothèse, Méthode du noyau, Modèles emboités. 


\title{
Nonparametric Significance Testing
}

\author{
By Pascal Lavergne and Quang H. Vuong
}

\section{Introduction}

In recent years, considerable work has been devoted to testing a parametric regression model against a semi or a nonparametric alternative. An approach that has attracted a lot of attention relies on smoothing techniques and compares the parametric fit with a smooth nonparametric one. Examples include Cleveland and Devlin (1988), Eubank and Spiegelman (1990), Eubank and Hart (1993), Gozalo (1993), Härdle and Mammen (1993), Chen (1994), Horowitz and Härdle (1994), Hong and White (1995), Zheng (1996) among others. ${ }^{1}$

In contrast, the issue of testing a nonparametric null against a nonparametric alternative has attracted less attention. A leading case where such a situation naturally arises is testing the significance of some explanatory variables in a regression function. Well-known procedures have been proposed in parametric settings, but their outcomes crucially depend on the choice of the parametric specification. When it is not desirable to adopt a finite parameterization, nonparametric regression provides a suitable alternative. A special case that has been investigated in several previous papers is the problem of testing for no effect. To our knowledge, the general case where the nonparametric null is nondegenerate has been considered only in a few published studies. Gozalo (1993) considers conditional moment tests which are made consistent against all alternatives through randomization. Yatchew (1992) considers the difference in residual sums of squares and

\footnotetext{
${ }^{1}$ Another approach uses empirical processes based on residuals of the parametric model, see Bierens (1982, 1990), Diebolt (1995) and Stute (1997). It seems however difficult to extend the empirical process approach to the situation considered in this paper.
} 
uses sample splitting to circumvent its well-known $\sqrt{n}$-degeneracy in a nested situation, while Lavergne and Vuong (1996) treat the nonnested case. ${ }^{2}$

Our objective is to propose a testing procedure for the significance of a subset of explanatory continuous variables in a nonparametric regression, which circumvents the drawbacks of previously proposed ones. Namely, our procedure does not use randomization but is nevertheless consistent against any deviation from the null hypothesis of no effect of the variables under test. Instead of using sample splitting or weighting, we deal with the $\sqrt{n}$-degeneracy issue to obtain a test statistic with a faster rate than $\sqrt{n}$. Our test statistic is based on the kernel method. We characterize its asymptotic distribution not only under the null hypothesis but also under a sequence of local alternatives. Our assumptions do not require normality or homoscedasticity of the regression errors and are not much more demanding on the bandwidths and on the considered functions than in nonparametric estimation. Though our test statistic is similar in spirit to that recently proposed by Fan and Li (1996), we require less restrictive theoretical conditions on the smoothing parameters. As a result, our testing procedure does not require oversmoothing of the null regression model relative to the alternative one and hence puts both models on equal footing. In small samples, our simulation results show that our test statistic is nearly unbiased under the null hypothesis and leads to a test that is more powerful than Fan and Li's one under a wide spectrum of alternatives.

The paper is organised as follows. In Section 2, we present our test statistic and we study its asymptotic properties under a sequence of local alternatives. We also show how our framework can accomodate the special case of testing the joint significance of all the regressors. Section 3 studies the small sample behavior of our testing procedure by means of Monte-Carlo experiments and compares it with the one proposed by Fan and Li (1996). All the proofs are relegated to Section 4.

\footnotetext{
${ }^{2}$ Unpublished related work includes Ait-Sahalia, Bickel and Stoker (1994) and Gozalo (1995).
} 


\section{Theoretical Results}

Suppose $\left(X_{2 i}, Y_{i}\right), i=1, \ldots, n$, is a random sample from a $\left(p_{2}+1\right)$-variate distribution of $\left(X_{2}, Y\right)$, and let $X_{1} \subset X_{2}$ be a $p_{1}$-vector, $0<p_{1}<p_{2}$. Throughout we denote densities of $X_{1}$ and $X_{2}$ by $f_{1}(\cdot)$ and $f_{2}(\cdot)$. Let $E\left[Y \mid X_{1}\right]=r_{1}\left(X_{1}\right)$ and $E\left[Y \mid X_{2}\right]=r_{2}\left(X_{2}\right)$. The null hypothesis of interest is $H_{0}: r_{1}\left(X_{1}\right)=r_{2}\left(X_{2}\right)$ a.s., or equivalently, $H_{0}: E\left[u_{1} \mid X_{2}\right]=0$ a.s., where $u_{1}=Y-r_{1}\left(X_{1}\right)$. Our procedure can be viewed as a test of the unconditional moment restriction $E\left[u_{1} \Psi\left(X_{2}\right)\right]=0$, with $\Psi\left(X_{2}\right)=E\left(u_{1} \mid X_{2}\right) f_{1}^{2}\left(X_{1}\right) f_{2}\left(X_{2}\right)$. Indeed, this particular choice makes the test consistent against any alternative to $H_{0}$ as

$$
\begin{aligned}
E\left[u_{1} E\left(u_{1} \mid X_{2}\right) f_{1}^{2}\left(X_{1}\right) f_{2}\left(X_{2}\right)\right] & =E\left[E^{2}\left(u_{1} \mid X_{2}\right) f_{1}^{2}\left(X_{1}\right) f_{2}\left(X_{2}\right)\right] \\
& =E\left[\left(r_{2}\left(X_{2}\right)-r_{1}\left(X_{1}\right)\right)^{2} f_{1}^{2}\left(X_{1}\right) f_{2}\left(X_{2}\right)\right]
\end{aligned}
$$

Let $K$ and $L$ be two kernels on $\mathbb{I R}^{p_{2}}$ and $\mathbb{R}^{p_{1}}$, respectively, and let $h$ and $g$ be two bandwidths. To test $H_{0}$, we consider

$$
V_{n}=\frac{1}{n^{(4)}} \sum_{a}\left(Y_{i}-Y_{k}\right)\left(Y_{j}-Y_{l}\right) L_{n i k} L_{n j l} K_{n i j}
$$

where $\sum_{a}$ denotes summation over the arrangements of $m$ distinct elements $\left\{i_{1}, \ldots, i_{m}\right\}$ from $\{1, \ldots, n\}$ with $n^{(m)}=n ! /(n-m)$ ! the number of these arrangements, and where $L_{n i k} \equiv g^{-p_{1}} L\left[\left(X_{1 i}-X_{1 k}\right) / g\right]$ and $K_{n i j} \equiv h^{-p_{2}} K\left[\left(X_{2 i}-X_{2 j}\right) / h\right]$.

The statistic $V_{n}$ is simple to compute, and in particular does no require any trimming. It constitutes a natural basis for testing $H_{0}$, because it actually estimates $\left.E\left[u_{1} \Psi\left(X_{2}\right)\right)\right]$. Indeed, assuming that $u_{1 i} f_{1}\left(X_{1 i}\right)$ is observed, a sample analog of the latter is

$$
V_{0 n}=\frac{1}{n^{(2)}} \sum_{a} u_{1 i} f_{1}\left(X_{1 i}\right) u_{1 j} f_{1}\left(X_{1 j}\right) K_{n i j}
$$

Fan and Li (1996) obtain their statistic $I_{n}$ from $V_{0 n}$ by replacing $u_{1 i} f_{1}\left(X_{1 i}\right)$ by its leave-one out kernel estimate. While our test statistic resembles Fan and Li's one, it was derived independently and differs from theirs by some important terms. ${ }^{3}$ Specifically,

$$
n^{(4)} V_{n}=n(n-1)^{3} I_{n}-n^{(3)} V_{1 n}-2 n^{(3)} V_{2 n},
$$

\footnotetext{
${ }^{3}$ Fan and Li (1996) also impose that the two kernels $K(\cdot)$ and $L(\cdot)$ are product kernels with the same univariate kernel.
} 
with

$$
V_{1 n}=\frac{1}{n^{(3)}} \sum_{a}\left(Y_{i}-Y_{k}\right)\left(Y_{j}-Y_{k}\right) L_{n i k} L_{n j k} K_{n i j}
$$

and

$$
V_{2 n}=\frac{1}{n^{(3)}} \sum_{a}\left(Y_{i}-Y_{j}\right)\left(Y_{j}-Y_{k}\right) L_{n i j} L_{n j k} K_{n i j}
$$

In effect, our statistic $V_{n}$ removes all "diagonal" terms from $I_{n}$, thus reducing the bias of the statistic without altering its properties as a test statistic for $H_{0}$. A similar idea has been recently proposed by Heffernan (1997) for unbiased estimation of central moments by $U$-statistics. In small samples, the bias reduction can be substantial, as our Monte-Carlo study of Section 3 shows. In practice, one may use the above formula instead of (2.1) to compute $V_{n}$.

To study the behavior of $V_{n}$ under the null and some local alternative hypotheses simultaneously, we write

$$
H_{1 n}: r_{2}\left(X_{2}\right)=r_{1}\left(X_{1}\right)+\delta_{n} d\left(X_{2}\right) \text {, with } \delta_{n} \in[0,1] \text {. }
$$

We let $d\left(X_{2}\right) \equiv 0$ if $\delta_{n}=0$. This general formulation allows us to include local alternatives, whose rates of convergence to $H_{0}$ are given by $\delta_{n}$. We need the following definitions and assumptions.

Definition 1 : (i) $\mathcal{U}^{p}$ is the class of integrable uniformly continuous functions from $\mathbb{I R}^{p}$ to IR. (ii) $\mathcal{D}_{m, q}^{p}$ is the class of $m$-times differentiable functions from $\mathbb{I}^{p}$ to $\mathbb{R}$, with derivatives of order $m$ that are uniformly Lipschitz continuous of order $q$.

Definition $2: \mathcal{K}_{m}^{p}, m \geq 2$, is the class of even integrable functions $K: \mathbb{I}^{p} \rightarrow \mathbb{I R}$ with compact support satisfying $\int K(s) d s=1$ and

$$
\int s_{1}^{\alpha_{1}} \ldots s_{p}^{\alpha_{p}} K(s) d s=0 \quad \text { for } 0<\sum_{i=1}^{p} \alpha_{i} \leq m-1 .
$$

Assumption $1:\left\{\left(X_{2 i}, Y_{i}\right), i=1, \ldots, n\right\}$ is an i.i.d. sample from an absolutely continuous (with respect to Lebesgue measure) $\left(p_{2}+1\right)$-variate distribution, and $E\left[Y^{8}\right]<\infty$.

Assumption 2 : (i) $f_{1}\left(X_{1}\right)$ and $r_{1}\left(X_{1}\right) f_{1}\left(X_{1}\right)$ belong to $\mathcal{U}^{p_{1}} \cap \mathcal{D}_{m_{1}, q_{1}}^{p_{1}}, m_{1} \geq 2$, and also $E\left(u_{1}^{2} \mid X_{1}\right) f_{1}\left(X_{1}\right)$ belongs to $\mathcal{U}^{p_{1}}$. (ii) $f_{2}\left(X_{2}\right), r_{2}\left(X_{2}\right) f_{1}\left(X_{1}\right) f_{2}\left(X_{2}\right), E\left(u_{1}^{2} \mid X_{2}\right) f_{1}^{2}\left(X_{1}\right) f_{2}\left(X_{2}\right)$ and $E\left(u_{1}^{4} \mid X_{2}\right) f_{1}^{4}\left(X_{1}\right) f_{2}\left(X_{2}\right)$ belong to $\mathcal{U}^{p_{2}}$. (iii) $K(\cdot) \in \mathcal{K}_{2}^{p_{2}}$ and $L(\cdot) \in \mathcal{K}_{m_{1}}^{p_{1}}$. 
Theorem 1 : Under Assumptions 1-D, if $h \rightarrow 0, g \rightarrow 0, n h^{p_{2}} \rightarrow+\infty, n g^{p_{1}} \rightarrow \infty$, $h^{p_{2}} / g^{p_{1}} \rightarrow 0$ and $n h^{p_{2} / 2} g^{2\left(m_{1}+q_{1}\right)} \rightarrow 0$, then as $n \rightarrow \infty$,

(i) $n h^{p_{2} / 2} V_{n} \stackrel{d}{\longrightarrow} N\left(C \mu, \omega^{2}\right) \quad$ if $\delta_{n}^{2} n h^{p_{2} / 2} \rightarrow C<\infty$,

(ii) $n h^{p_{2} / 2} V_{n} \stackrel{p}{\rightarrow}+\infty \quad$ if $\delta_{n}^{2} n h^{p_{2} / 2} \rightarrow \infty$,

where $\mu=E\left[d^{2}\left(X_{2}\right) f_{1}^{2}\left(X_{1}\right) f_{2}\left(X_{2}\right)\right]$ and $\omega^{2}=2 E\left[E^{2}\left(u_{1}^{2} \mid X_{2}\right) f_{1}^{4}\left(X_{1}\right) f_{2}\left(X_{2}\right)\right] \int K^{2}(s) d s$.

Remark 1: As shown in Section 4, $V_{n}$ has the same behavior as $V_{0 n}$. In general, $V_{0 n}$ is such that $\sqrt{n}\left[V_{0 n}-E\left(V_{0 n}\right)\right]$ converges to a normal distribution $N\left(0, \tau^{2}\right)$, where $\tau^{2}$ is the semiparametric efficiency bound for estimating $E\left[u_{1} \Psi\left(X_{2}\right)\right]$. But under $H_{0}$, we have both $E\left(V_{0 n}\right)=0$ and $\tau^{2}=0$. This degeneracy leads us to consider higher-order terms in the expansion of $V_{0 n}$. For this we use a central limit theorem for degenerate U-statistics, see Hall $(1984 \mathrm{a}){ }^{4}$

Remark 2: Assumption 2 requires smoothness conditions on the underlying functions and kernels that are standard in nonparametric estimation. Functions of $X_{1}$ are assumed to be at least as smooth as functions of $X_{2}$. This is compatible with the nested situation under consideration. Instead, Fan and Li (1996) requires similar smoothness of the constrained and unconstrained regression functions.

Remark 3: The generalization of our test to the situation where some of the $X_{1}$ are discrete with finite support is straightforward, as discrete variables neither create any bias nor change the variance of the nonparametric estimators. Our general results are not affected, where bandwidths only apply to continuous regressors. In particular, when all regressors $X_{1}$ are discrete and all regressors under test are continuous, our assumptions on the bandwidths reduce to the usual ones, i.e. $h \rightarrow 0$ and $n h^{p_{2} / 2} \rightarrow+\infty$.

Remark 4: One of the main problems in obtaining asymptotic distributions of semiparametric estimators is the relative vanishing rates of the bias and variance terms from nonparametric estimation. For instance, Samarov (1993) notes that the bias term may dominate the variance term for his test statistic. Hall (1984a,1984b) finds that the squared bias term of the integrated square error of kernel estimators is of order $h^{4}$. In the context of parametric specification testing, Hong (1993) and Gozalo (1995) find a bias term of

\footnotetext{
${ }^{4}$ As we consider local alternatives and a finer decomposition of $V_{n}$, this prevents us to use Fan and Li's proofs.
} 
order $h^{2}$ and proposes a statistic that balances it with the variance term. In our context, the bias problem arises in each of the two smoothing steps: the nonparametric regression of $Y$ on $X_{1}$ and the projection of the residual $u_{1}$ on $X_{2}$. The form of our statistic eliminates the bias in the second step, so that $E\left(V_{0 n}\right)=0$ under $H_{0}$. On the other hand, the bias from the first step is controlled through the "bias" condition $n h^{p_{2} / 2} g^{2\left(m_{1}+q_{1}\right)} \rightarrow 0$, as in Fan and Li (1996).

Remark 5: Though the theory is developped for a generic bandwidth ( $g$ or $h$ ) in each step, it is straighforward to extend it to a vanishing individual bandwidth for each regressor in each step. ${ }^{5}$ In this case, one should replace $g^{p_{1}}$ and $h^{p_{2}}$ by $g_{1} g_{2} \ldots g_{p_{1}}$ and $h_{1} h_{2} \ldots h_{p_{2}}$, respectively. The "bias" condition becomes $n \prod_{i=1}^{p_{2}} h_{i}^{1 / 2}\left[\max _{i=1, \ldots, p_{1}} g_{i}\right]^{2\left(m_{1}+q_{1}\right)} \rightarrow 0$.

Remark 6: Our assumptions on the bandwidths include the usual ones. The condition on the ratio $h^{p_{2}} / g^{p_{1}}$ means that the variance of nonparametric estimators in the model with $p_{1}$ regressors is smaller than the variance of nonparametric estimators in the complete model. This seems reasonable in view of the higher sparsity of the data in high dimensional spaces, leading to the well-known "curse of dimensionality." In our testing framework, this condition can be better understood by considering individual bandwidths. In this case, it seems natural to use individual bandwidths for the regressors $X_{1}$ not under test that are identical between both steps, namely $g_{i}=h_{i}, i=1, \ldots p_{1}$, to avoid incorrect rejection of the null hypothesis. Then our ratio condition reduces to $\prod_{i=p_{1}+1}^{p_{2}} h_{i} \rightarrow 0$. This is no longer restrictive as vanishing individual bandwidths are obviously necessary to obtain a consistent test. Hence our "ratio" condition on the relative rates of the bandwidths seems to be minimal for testing the significance of continuous regressors. In contrast, when the regressors under test are discrete, $\prod_{i=p_{1}+1}^{p_{2}} h_{i}$ needs not vanish, so that restricted and nonrestricted nonparametric estimates jointly determine the limit distribution of the test statistic, as studied by Lavergne (1997).

Fan and $\mathrm{Li}$ (1996) requires the stronger condition $h^{p_{2}} / g^{2 p_{1}} \rightarrow 0$. As a result, Fan and Li's testing procedure excludes a large domain of bandwidths, including the optimal bandwidth rates for estimation $n^{-1 /[p+2(m+q)]}$ when the dimension of $X_{1}$ is close to the dimension of $X_{2}$, and this for any degree of smoothness in the underlying regressions. For instance, this arises when $p_{2}=2$ and $p_{1}=1$, or $p_{2}=3$ and $p_{1}=2$. In contrast, our testing

\footnotetext{
${ }^{5}$ One could also consider a more general form of kernel estimators as in Robinson (1983).
} 
procedure allows for a broader choice, including the optimal estimation rates when the constrained regression is sufficiently smooth, though these optimal estimation rates need not be optimal for testing purposes, see Guerre and Lavergne (1998).

The asymptotic variance $\omega^{2}$ can be written as

$$
2 E\left[\left(u_{1} f_{1}\left(X_{1}\right)\right)^{2} E\left[\left(u_{1} f_{1}\left(X_{1}\right)\right)^{2} \mid X_{2}\right] f_{2}\left(X_{2}\right)\right] \int K^{2}(s) d s .
$$

It depends on the kernel through $\int K^{2}(s) d s$. This quantity can be minimized in the class of product nonnegative even kernels by choosing the Epanechnikov kernel, see Epanechnikov (1969). Following (2.1), an estimator of $\omega^{2}$ is

$$
\omega_{n}^{2}=\frac{2}{n^{(6)}} \sum_{a}\left(Y_{i}-Y_{k}\right)\left(Y_{i}-Y_{k^{\prime}}\right)\left(Y_{j}-Y_{l}\right)\left(Y_{j}-Y_{l^{\prime}}\right) L_{n i k} L_{n i k^{\prime}} L_{n j l} L_{n j l^{\prime}} h^{p_{2}} K_{n i j}^{2}
$$

An alternative estimator, which is computationally less demanding, but more biased in small samples, is

$$
\omega_{n}^{2}=\frac{2}{n^{(2)}} \sum_{a} \widehat{u}_{1 i} \widehat{u}_{1 j} h^{p_{2}} K_{n i j}^{2},
$$

where $\hat{u}_{1 i}$ is the kernel estimator of the residual $u_{1 i}$. The consistency of either form of $\omega_{n}^{2}$ is shown using similar arguments as in Theorem 1's proof. Therefore, we can propose $n h^{p_{2} / 2} V_{n} / \omega_{n}$ as a test statistic for $H_{0}$. From Theorem 1 , by letting $\delta_{n}=0$ or 1 , this test statistic is asymptotically $N(0,1)$ under $H_{0}$ and diverges to $+\infty$ against any fixed alternative to $H_{0}$. The test is therefore a one-sided normal test. Moreover, the test has power to detect local alternatives $H_{1 n}$ approaching the null at rate slower than $\left(\sqrt{n} h^{p_{2} / 4}\right)^{-1}$. This rate agrees with that found in parametric specification testing procedures that use smoothing. ${ }^{6}$

Although Theorem 1 suggests that suitable critical values for our testing procedure can be obtained from the standard normal distribution, results from Eubank and LaRiccia (1993) and Härdle and Mammen (1993) among others indicate that the normal approximation may not be adequate for small sample sizes. Indeed, our test statistic behaves like a weighted sum of chi-squares, in an asymptotic sense, and accordingly may approach

\footnotetext{
${ }^{6}$ It is always possible to modify our test statistic and make it consistent against some chosen local $\sqrt{n}$-alternatives. This is done by adding to our test statistic a suitable $M$-test statistic based on the estimated residuals and by deriving the resulting limiting distribution.
} 
normality slowly, especially for high dimensional settings. One alternative is to use a $\chi^{2}$ approximation, as proposed by Hall (1983) and Buckley and Eagleson (1988) and used by Eubank and LaRiccia (1993) and Chen (1994) in the context of parametric specification testing. While such a correction may help in high dimensions, it did not prove much useful in our limited Monte-Carlo experiments, where the normal approximation seems to work well. Another alternative is to use resampling techniques, such as the wild bootstrap considered by Härdle and Mammen (1993). The theoretical justification of such a technique in our context, and specifically the conditions under which it applies, is left for further research.

Lastly, it is possible to extend our procedure to the case where $p_{1}=0$, i.e. testing for no effect of all the regressors $X_{2}$. In this case the null hypothesis of interest is $H_{0}^{*}$ : $r_{2}\left(X_{2}\right)=E(Y)$ a.s. To test $H_{0}^{*}$, we can readily modify $(2.1)$ to get

$$
V_{n}^{*}=\frac{1}{n^{(4)}} \sum_{a}\left(Y_{i}-Y_{k}\right)\left(Y_{j}-Y_{l}\right) K_{n i j}
$$

As before, we consider the local alternatives $r_{2}\left(X_{2}\right)=E(Y)+\delta_{n} d\left(X_{2}\right)$, with $\delta_{n} \in[0,1]$. Our Assumption 2 now reduces to the usual one in nonparametric estimation, namely

Assumption 3 (i) $f_{2}\left(X_{2}\right), r_{2}\left(X_{2}\right) f_{2}\left(X_{2}\right), \operatorname{Var}^{2}\left(Y \mid X_{2}\right) f_{2}^{2}\left(X_{2}\right)$ and $E\left((Y-c)^{4} \mid X_{2}\right) f_{2}\left(X_{2}\right)$ belong to $\mathcal{U}^{p_{2}}$. (iii) $K \in \mathcal{K}_{2}^{p_{2}}$.

Corollary 1 : Under Assumptions 1 and 3 , if $h \rightarrow 0, n h^{p_{2}} \rightarrow+\infty$, then as $n \rightarrow \infty$,

$$
\begin{array}{lll}
\text { (i) } n h^{p_{2} / 2} V_{n}^{*} \stackrel{d}{\longrightarrow} N\left(C \mu^{*}, \omega^{* 2}\right) & \text { if } \delta_{n}^{2} n h^{p_{2} / 2} \rightarrow C<\infty, \\
\text { (ii) } n h^{p_{2} / 2} V_{n}^{*} \stackrel{p}{\longrightarrow}+\infty & \text { if } \delta_{n}^{2} n h^{p_{2} / 2} \rightarrow \infty,
\end{array}
$$

where $\mu^{*}=E\left[d^{2}\left(X_{2}\right) f_{2}\left(X_{2}\right)\right]$ and $\omega^{* 2}=2 E\left[\operatorname{Var}^{2}\left(Y \mid X_{2}\right) f_{2}\left(X_{2}\right)\right] \int K^{2}(s) d s$.

Similarly to $\omega_{n}^{2}$, a consistent estimator of $\omega^{* 2}$ is

$$
\omega_{n}^{* 2}=\frac{2}{n^{(6)}} \sum_{a}\left(Y_{i}-Y_{k}\right)\left(Y_{i}-Y_{k^{\prime}}\right)\left(Y_{j}-Y_{l}\right)\left(Y_{j}-Y_{l^{\prime}}\right) h^{p_{2}} K_{n i j}^{2} .
$$

A consistent one-sided normal test for no effect of $X_{2}$ in the regression of $Y$ can thus be based on $n h^{p_{2} / 2} V_{n}^{*} / \omega_{n}^{*}$. As before, this test has power to detect local alternatives approaching the null at rate slower than $\left(\sqrt{n} h^{p_{2} / 4}\right)^{-1}$. 
Many other tests have been previously proposed for the special case of testing for no effect, as reviewed in Hart's (1997) monograph. Because the null is very simple in this case, it is possible to apply the empirical process approach and to derive omnibus tests, see e.g. Buckley (1991) and Bierens (1982, 1990). Alternatively, tests based on smoothing ideas have been considered, see e.g. Eubank and Hart (1993). In particular, it is possible to allow for data-driven smoothing parameters in such tests, see Barry and Hartigan (1990), or to construct a test based on the smoothing parameter itself, see Eubank and Hart (1992). However, with the exception of Bierens $(1982,1990)$, all these tests have been developed in the special case of a single regressor and homoscedastic errors. In addition, the limiting behavior of some of these tests is nonstandard.

The statistic $V_{n}^{*}$ resembles Zheng's (1996) statistic for parametric specification testing in the case where the parametric model reduces to the constant regression, but removes all "diagonal" terms from the latter to make it unbiased under the null. Indeed, because the estimation of the smallest regression model is actually parametric, there is no bias corresponding to this stage. As the form of our statistic also eliminates the bias in the second stage, a notable feature of our statistic is that it is unbiased under the null hypothesis, i.e. $E\left(V_{n}^{*}\right)=0$ under $H_{0}$. This is especially valuable in small samples.

\section{Monte-Carlo Study}

In this section, we investigate the small sample behavior of our test and study its performances relative to Fan and Li's (1996) test, hereafter FL test. We generate data through

$$
Y=a X_{1}+b X_{1}^{3}+d(W)+U
$$

where $X_{1}$ and $W$ are independent and distributed as $N(0,1)$ and $U$ is independently distributed of the regressors as $N\left(0, \sigma^{2}\right)$. The null hypothesis corresponds to $d(W) \equiv$ 0 , and we consider different forms of alternatives as specified by $d(\cdot)$. We impose the restriction that $E[d(W)]=0$, so that the nonparametric regression $r_{1}\left(X_{1}\right)$ remains the same whatever the data generating process. We set the parameters $a, b$ and $\sigma^{2}$ to $-1,1$ and 4 respectively, so that the part of the variance of $Y$ explained in its nonparametric regression on $X_{1}$ is moderate, i.e. $71 \%$. 
We consider small $(n=100)$ and moderate $(n=200)$ sample sizes and run 2000 replications. We choose $K(\cdot)$ and $L(\cdot)$ as product kernels of the univariate Epanechnikov kernel with support $[-1,1]$, i.e. $L(u)=(3 / 4)\left(1-u^{2}\right) \mathbb{I I}[|u| \leq 1]$. As indicated in Remark 5 , we can use individual bandwidths. The bandwidth parameter for the restricted model is chosen as $g=\hat{s}_{X_{1}} n^{-1 / 5}$, where $\hat{s}_{X_{1}}$ is the estimated standard deviation of $X_{1}$. This corresponds to the usual rule-of-thumb in kernel estimation, see e.g. Härdle (1991). For the unrestricted model, we keep the same smoothing parameter as in the restricted one for the first dimension, i.e. $X_{1}$, and choose the parameter for the second dimension, i.e. $Z$, as $h_{2}=c \hat{s}_{W} n^{-1 / 5}$, where $\hat{s}_{W}$ is the estimated standard deviation of $W$. Keeping the same bandwidth for regressors that are common to both models, in our case $X_{1}$, seems a natural choice in our testing framework. For the regressors under test, i.e. $W$, we apply the same rule-of-thumb with an additional varying constant $c$ to investigate the sensitivity of our results to the smoothing parameter's choice. ${ }^{7}$

The design of the alternatives has been chosen to investigate the power of the competing tests with respect to the magnitude and the frequency of $d(\cdot)$. For the magnitude, we consider three linear alternatives of the form

$$
d(W)=\alpha W
$$

with $\alpha=0.5,1$ and 2 corresponding respectively to $D G P_{1}, D G P_{2}$ and $D G P_{3}$. This allows us to compare the performances of the nonparametric tests to the standard Fisher test based on the true Model (3.3). Alternatives corresponding to varying frequencies are defined through

$$
d(W)=\sin (\delta \pi W)
$$

with $\delta=2,1,2 / 3$ and $1 / 2$ corresponding respectively to $D G P_{4}, D G P_{5}, D G P_{6}$ and $D G P_{7}$. These departures from the null are of special interest, as it is known that smoothing tests of parametric specifications are sensitive to the frequency of the alternatives, see Eubank and Hart (1993), Kuchibhatla and Hart (1996) and Hart (1997). We expect that such a feature will hold for nonparametric significance tests.

Table 1 reports our Monte-Carlo results for the null hypothesis $\left(D G P_{0}\right)$ and the linear alternatives. For each sample size $(n=100,200)$, we let the constant $c$ be $0.25,0.5,1,2,4$.

\footnotetext{
${ }^{7}$ In our setup, we have $m_{1}=2$ and $q_{1}=1$, so that our bandwidths satisfy Theorem 1's conditions.
} 
For each case, the first and second rows give the mean with standard deviation in parentheses of our test and FL test, respectively. For computational reasons, we use the simplest, but biased, estimator of the variance (2.2). The third and fourth rows give empirical levels of rejections for our test and FL test. The first figure corresponds to a $5 \%$ nominal level, while the second one corresponds to a $10 \%$ nominal level. For each sample size, the last row reports empirical rejection rates of the F-test for the same nominal levels.

The first column relates to the null hypothesis. First, the mean of our test statistic is very close to zero, i.e our test statistic is nearly unbiased, irrespective of the smoothing parameter, see Remark 3. This is in sharp contrast with FL test statistic, which is always negatively biased, up to -1.15 . Second, the standard deviations of both test statistics are smaller than one. This is due partly to the fact that the simple variance estimator (2.2) always overestimates the variance. Although both tests exhibit empirical sizes that are smaller than the nominal ones, FL test can be considerably undersized due to its strong negative bias. The size of our test is much closer to its nominal size, especially for bandwidths that are somewhat smaller than the rule-of-thumb. The empirical level as a function of the bandwidth $h_{2}$ displays an inverse $U$-shape, as for very small bandwidths $h_{2}$, our statistic is identically zero.

Regarding the linear alternatives, we find that FL test statistic is more variable than ours. Moreover, our test statistic has a higher mean than FL one, which is due to the negative bias of the latter. This leads to a systematic higher empirical power for our test. As expected, power is increasing with the magnitude of the departure from the null, as measured by $\alpha$. Our test can detect small linear alternatives such as $D G P_{1}$, unlike FL test which has close to trivial power in this situation. Furthermore, the power performance of our test can equal that of the Fisher test (see $D G P_{3}$ ), although the design is ideal for the latter. Our results also indicate that the highest power is attained for our test for the largest tried bandwidth, which is expected since the alternative is linear and the kernel smoother is a straight line for large bandwidths. However, using an infinite bandwidth should ultimately lead to a trivial power.

Table 2 has the same structure as Table 1 and reports results relative to the sinus alternatives. As in Table 1, our test statistic exhibits a larger mean and a smaller variance than FL one, and hence achieves higher power in all cases. The empirical power as a 
function of $h_{2}$ displays an inverse $U$-shape for both tests. As shown in Figure 1, our test uniformly dominates FL test for a large range of bandwidths. The maximum power of our test can be up to fifty percent higher. It is achieved for a bandwidth that increases with the smoothness of the alternative, as could be expected. Hence, our results suggest that the bandwidth should be adapted to the frequency of the alternative, namely the higher the frequency, the smaller the bandwidth should be.

For comparative purpose, we also provide the empirical rejection rates of the F-test assuming a linear specification in $W$. The lowest frequency alternative $D G P_{7}$ is close to a linear specification in the range $[-1,1]$. Given that $W$ is $N(0,1)$, the F-test therefore performs quite well, while our test has acceptable power up to $78 \%$. For high frequency alternatives $D G P_{4}$ and $D G P_{5}$, the F-test has trivial power irrespective of sample size, while our test can attain an empirical power of $50 \%$ or $68 \%$ respectively for a moderate sample size of 200 .

To sum up, our test has better size and power than FL test in all cases and seems to exhibit good properties for a wide range of nonlinear alternatives. Our Monte-Carlo study points out the importance of the bandwidth choice. There is clearly a trade-off between size and power. A better sized test seems to be achieved by slight undersmoothing relative to the rule-of-thumb, while better power is obtained in most cases by oversmoothing of the variable under test. Our limited experiments suggest that the usual rule-of-thumb $(c=1)$ leads to an acceptable compromise between size and power.

\section{Proofs}

Notations: In what follows, $f_{i} \equiv f_{1}\left(X_{1 i}\right), f_{2 i} \equiv f_{2}\left(X_{2 i}\right), r_{i} \equiv r_{1}\left(X_{1 i}\right), r_{2 i} \equiv r_{2}\left(X_{2 i}\right)$, $u_{i} \equiv Y_{i}-r_{i}, u_{2 i} \equiv Y_{i}-r_{2 i}, d_{i} \equiv d\left(X_{2 i}\right)$ and $Z_{i}$ stands for $\left(Y_{i}, X_{2 i}\right), i=0,1, \ldots, n$. Also $\boldsymbol{K} \equiv|K|$ and $\boldsymbol{L} \equiv|L|$ and $i, j, k, l, i^{\prime}, j^{\prime}, k^{\prime}, l^{\prime}$ refer to indices that are pairwise different unless stated otherwise. We let $\hat{f}_{i}=(n-1)^{-1} \sum_{k \neq i} L_{n i k}$, and more generally for any index set $I$ not containing $i$ with cardinality $|I|, \hat{f}_{i}^{I}=(n-1-|I|)^{-1} \sum_{k \neq i, k \notin I} L_{n i k}$. 


\subsection{Proof of Theorem 1}

As $Y_{i}-Y_{k}=\left(u_{i}-u_{k}\right)+\left(r_{i}-r_{k}\right)$, and as $K$ is even, we have from (2.1)

$$
\begin{aligned}
V_{n}= & \frac{1}{n^{(4)}} \sum_{a}\left(u_{i}-u_{k}\right)\left(u_{j}-u_{l}\right) L_{n i k} L_{n j l} K_{n i j}+\frac{2}{n^{(4)}} \sum_{a}\left(u_{i}-u_{k}\right)\left(r_{j}-r_{l}\right) L_{n i k} L_{n j l} K_{n i j} \\
& +\frac{1}{n^{(4)}} \sum_{a}\left(r_{i}-r_{k}\right)\left(r_{j}-r_{l}\right) L_{n i k} L_{n j l} K_{n i j}=I_{1}+2 I_{2}+I_{3}, \\
\text { where } I_{1}= & \frac{n-2}{n-3} \frac{1}{n^{(2)}} \sum_{a} u_{i} u_{j} f_{i} f_{j} K_{n i j}+\frac{2(n-2)}{n-3} \frac{1}{n^{(2)}} \sum_{a} u_{i}\left(\hat{f}_{i}^{j}-f_{i}\right) u_{j} f_{j} K_{n i j} \\
& +\frac{n-2}{n-3} \frac{1}{n^{(2)}} \sum_{a} u_{i}\left(\hat{f}_{i}^{j}-f_{i}\right) u_{j}\left(\hat{f}_{j}^{i}-f_{j}\right) K_{n i j}-\frac{2}{n^{(3)}} \sum_{a} u_{i} f_{i} u_{l} L_{n j l} K_{n i j} \\
& -\frac{2}{n^{(3)}} \sum_{a} u_{i}\left(\hat{f}_{i}^{j, l}-f_{i}\right) u_{l} L_{n j l} K_{n i j}+\frac{1}{n^{(4)}} \sum_{a} u_{k} u_{l} L_{n i k} L_{n j l} K_{n i j} \\
& -\frac{1}{n^{(4)}} \sum_{a} u_{i} u_{j} L_{n i k} L_{n j k} K_{n i j} \\
& \frac{n-2}{n-3}\left[V_{0 n}+2 I_{1,1}+I_{1,2}\right]-2 I_{1,3}-2 I_{1,4}+I_{1,5}-I_{1,6}, \\
I_{2}= & \frac{1}{n^{(3)}} \sum_{a} u_{i} f_{i}\left(r_{j}-r_{l}\right) L_{n j l} K_{n i j}+\frac{1}{n^{(3)}} \sum_{a} u_{i}\left(\hat{f}_{i}^{j, l}-f_{i}\right)\left(r_{j}-r_{l}\right) L_{n j l} K_{n i j} \\
& -\frac{1}{n^{(4)}} \sum_{a} u_{k}\left(r_{j}-r_{l}\right) L_{n i k} L_{n j l} K_{n i j}=I_{2,1}+I_{2,2}-I_{2,3} .
\end{aligned}
$$

Propositions 1 to 11 study each of the above terms. Collecting results, it follows that

$$
\begin{gathered}
n h^{p_{2} / 2} V_{0 n}=A_{n}+\delta_{n}^{2} n h^{p_{2} / 2} \mu_{n}+\delta_{n} \sqrt{n} h^{p_{2} / 2} O_{p}(1), \\
n h^{p_{2} / 2}\left[I_{1}-V_{0 n}\right]=\delta_{n}^{2} n h^{p_{2} / 2} o_{p}(1)+\delta_{n} \sqrt{n} h^{p_{2} / 2} O_{p}(1)+o_{p}(1), \\
n h^{p_{2} / 2} I_{2}=\delta_{n}^{2} n h^{p_{2} / 2} o_{p}(1)+\delta_{n} \sqrt{n} h^{p_{2} / 2} o_{p}(1)+\delta_{n} n h^{p_{2} / 2} g^{\left(m_{1}+q_{1}\right)} O_{p}(1)+o_{p}(1), \\
n h^{p_{2} / 2} I_{3}=o_{p}(1),
\end{gathered}
$$

where $A_{n} \stackrel{d}{\longrightarrow} N\left(0, \omega^{2}\right)$. Therefore

$$
n h^{p_{2} / 2}\left[V_{n}-V_{0 n}\right]=\delta_{n}^{2} n h^{p_{2} / 2} o_{p}(1)+\delta_{n} \sqrt{n} h^{p_{2} / 2} O_{p}(1)+\delta_{n} n h^{p_{2} / 2} g^{\left(m_{1}+q_{1}\right)} O_{p}(1)+o_{p}(1) .
$$

In case (i), $n h^{p_{2} / 2}\left[V_{n}-V_{0 n}\right]=o_{p}(1)$ and $n h^{p_{2} / 2} V_{0 n} \stackrel{d}{\longrightarrow} N\left(C \mu, \omega^{2}\right)$, as

$$
\begin{aligned}
\delta_{n} \sqrt{n} h^{p_{2} / 2} & =\left(\delta_{n}^{2} n h^{p_{2} / 2}\right)^{1 / 2} h^{p_{2} / 4}=o(1), \\
\text { and } \delta_{n} n h^{p_{2} / 2} g^{\left(m_{1}+q_{1}\right)} & =\left(\delta_{n}^{2} n h^{p_{2} / 2}\right)^{1 / 2}\left(n h^{p_{2} / 2} g^{2\left(m_{1}+q_{1}\right)}\right)^{1 / 2}=o(1) .
\end{aligned}
$$


In case (ii), $n h^{p_{2} / 2}\left[V_{n}-V_{0 n}\right]=o_{p}\left(\delta_{n}^{2} n h^{p_{2} / 2}\right)$ and $n h^{p_{2} / 2} V_{0 n}=\delta_{n}^{2} n h^{p_{2} / 2}\left[\mu_{n}+o_{p}(1)\right]$, as

$$
\begin{aligned}
\delta_{n} \sqrt{n} h^{p_{2} / 2} & =\left(\delta_{n}^{2} n h^{p_{2} / 2}\right) \frac{h^{p_{2} / 4}}{\left(\delta_{n}^{2} n h^{p_{2} / 2}\right)^{1 / 2}}=o\left(\delta_{n}^{2} n h^{p_{2} / 2}\right) \\
\text { and } \delta_{n} n h^{p_{2} / 2} g^{\left(m_{1}+q_{1}\right)} & =\left(\delta_{n}^{2} n h^{p_{2} / 2}\right) \frac{\left(n h^{p_{2} / 2} g^{2\left(m_{1}+q_{1}\right)}\right)^{1 / 2}}{\left(\delta_{n}^{2} n h^{p_{2} / 2}\right)^{1 / 2}}=o\left(\delta_{n}^{2} n h^{p_{2} / 2}\right) .
\end{aligned}
$$

Q.E.D.

\subsubsection{Distribution of $V_{0 n}$}

Proposition $1: n h^{p_{2} / 2} V_{0 n}=A_{n}+\delta_{n}^{2} n h^{p_{2} / 2} \mu_{n}+\delta_{n} \sqrt{n} h^{p_{2} / 2} B_{n}$,

where $\mu_{n} \longrightarrow \mu, A_{n} \stackrel{d}{\longrightarrow} N\left(0, \omega^{2}\right)$ and $B_{n} \stackrel{d}{\longrightarrow} 2 N\left(0, \xi-\delta^{2} \mu^{2}\right)$, with $\delta=\lim _{n \rightarrow \infty} \delta_{n}$ and $\xi=E\left[u_{1}^{2} d^{2}\left(X_{2}\right) f_{1}^{4}\left(X_{1}\right) f_{2}^{2}\left(X_{2}\right)\right]$.

Proof: Write $V_{0 n}=U_{0 n}+W_{0 n}-\theta_{n}$, where $H_{n}\left(Z_{i}, Z_{j}\right)=u_{i} u_{j} f_{i} f_{j} K_{n i j}, \theta_{n}=E\left[H_{n}\left(Z_{1}, Z_{0}\right)\right]$, $W_{0 n}=(2 / n) \sum_{i} E\left[H_{n}\left(Z_{i}, Z_{0}\right) \mid Z_{i}\right]$ and

$$
\begin{aligned}
U_{0 n} & =\left(\begin{array}{l}
n \\
2
\end{array}\right)^{-1} \sum_{i<j} \tilde{H}_{n}\left(Z_{i}, Z_{j}\right) \\
& =\left(\begin{array}{l}
n \\
2
\end{array}\right)^{-1} \sum_{i<j}\left\{H_{n}\left(Z_{i}, Z_{j}\right)-E\left[H_{n}\left(Z_{i}, Z_{0}\right) \mid Z_{i}\right]-E\left[H_{n}\left(Z_{0}, Z_{j}\right) \mid Z_{j}\right]+\theta_{n}\right\} .
\end{aligned}
$$

(i)

$$
\begin{aligned}
\theta_{n} & =E\left[u_{i} f_{i} u_{j} f_{j} K_{n i j}\right]=E\left[\left(u_{2 i}+\delta_{n} d_{i}\right) f_{i}\left(u_{2 j}+\delta_{n} d_{j}\right) f_{j} K_{n i j}\right] \\
& =\delta_{n}^{2} E\left[d_{i} f_{i} d_{j} f_{j} K_{n i j}\right]=\delta_{n}^{2} \mu_{n},
\end{aligned}
$$

with $\mu_{n} \longrightarrow \mu=E\left[d^{2}\left(X_{2}\right) f_{1}^{2}\left(X_{1}\right) f_{2}\left(X_{2}\right)\right]$, as $\delta_{n} d\left(X_{2}\right) f_{1}\left(X_{1}\right) f_{2}\left(X_{2}\right) \in \mathcal{U}^{p_{2}}$ and Lemma 1 . (ii) Distribution of $W_{0 n}$ :

$$
\begin{aligned}
E\left[E^{2}\left(H_{n}\left(Z_{i}, Z_{0}\right) \mid Z_{i}\right)\right] & =E\left[u_{i}^{2} f_{i}^{2} E^{2}\left(u_{0} f_{0} K_{n i 0} \mid Z_{i}\right)\right] \\
& =\delta_{n}^{2} E\left[u_{i}^{2} f_{i}^{2} E^{2}\left(d_{0} f_{0} K_{n i 0} \mid Z_{i}\right)\right]=\delta_{n}^{2} \xi_{n}
\end{aligned}
$$

with $\xi_{n} \longrightarrow \xi=E\left[u_{1}^{2} d^{2}\left(X_{2}\right) f_{1}^{4}\left(X_{1}\right) f_{2}^{2}\left(X_{2}\right)\right]$, as $\delta_{n} d\left(X_{2}\right) f_{1}\left(X_{1}\right) f_{2}\left(X_{2}\right) \in \mathcal{U}^{p_{2}}$ and Lemma 1. Now $E\left|E\left[H_{n}\left(Z_{i}, Z_{j}\right) \mid Z_{i}\right]\right|^{\nu}=E\left|u_{i}^{\nu} f_{i}^{\nu} E^{\nu}\left[u_{0} f_{0} K_{n i 0} \mid Z_{i}\right]\right|=O(1)=o\left(n^{\nu / 2-1}\right)$ for $2<\nu \leq 4$, as $E\left|Y^{2 \nu}\right|<\infty$. Thus, by Theorem 7.1 of Hoeffding (1948),

$$
\sqrt{n}\left[W_{0 n}-2 \theta_{n}\right] \rightarrow 2 \delta N\left(0, \xi-\delta^{2} \mu^{2}\right)
$$


(iii) Distribution of $U_{0 n}:$ As $E\left[\tilde{H}_{n}\left(Z_{i}, Z_{j}\right) \mid Z_{i}\right]=0$, by Theorem 1 of Hall (1984),

$$
n h^{p_{2} / 2} U_{0 n} \stackrel{d}{\longrightarrow} N\left(0, \omega^{2}\right) \quad \text { if } \quad \frac{E\left[\tilde{G}_{n}^{2}\right]+n^{-1} E\left[\tilde{H}_{n}^{4}\right]}{E^{2}\left[\tilde{H}_{n}^{2}\right]}=o(1),
$$

where $\tilde{G}_{n}\left(Z_{i}, Z_{j}\right)=E\left[\tilde{H}_{n}\left(Z_{i}, Z_{0}\right) \tilde{H}_{n}\left(Z_{j}, Z_{0}\right) \mid Z_{i}, Z_{j}\right]$ and $\omega^{2}=2 \lim _{n \rightarrow \infty} h^{p_{2}} E\left(\tilde{H}_{n}^{2}\right)$. By definition of $\tilde{H}_{n}\left(Z_{i}, Z_{j}\right)$, the above condition is equivalent to

$$
\frac{E\left[G_{n}^{2}\right]+n^{-1} E\left[H_{n}^{4}\right]}{E^{2}\left[H_{n}^{2}\right]}=o(1)
$$

where $G_{n}\left(Z_{i}, Z_{j}\right)=E\left[H_{n}\left(Z_{i}, Z_{0}\right) H_{n}\left(Z_{j}, Z_{0}\right) \mid Z_{i}, Z_{j}\right]$, and $\omega^{2}=2 \lim _{n \rightarrow \infty} h^{p_{2}} E\left(H_{n}^{2}\right)$.

Let $\sigma^{2}\left(X_{2}\right) \equiv E\left(u_{1}^{2} \mid X_{2}\right)$. As $\sigma^{2}\left(X_{2}\right) f_{1}^{2}\left(X_{1}\right) f_{2}\left(X_{2}\right) \in \mathcal{U}^{p_{2}}$, by Lemma 1 ,

$$
h^{p_{2}} E\left[H_{n}^{2}\left(Z_{i}, Z_{j}\right)\right]=h^{p_{2}} E\left[\sigma^{2}\left(X_{2 i}\right) \sigma^{2}\left(X_{2 j}\right) f_{i}^{2} f_{j}^{2} K_{n i j}^{2}\right] \longrightarrow \omega^{2} / 2,
$$

where $\omega^{2}=2 E\left[\sigma^{4}\left(X_{2}\right) f_{1}^{4}\left(X_{1}\right) f_{2}\left(X_{2}\right)\right] \int K^{2}(s) d s$.

As $E\left(u_{1}^{4} \mid X_{2}\right) f_{1}^{4}\left(X_{1}\right) f_{2}\left(X_{2}\right) \in \mathcal{U}^{p_{2}}$, by Lemma 1 ,

$$
E\left[H_{n}^{4}\right]=E\left[u_{i}^{4} u_{j}^{4} f_{i}^{4} f_{j}^{4} K_{n i j}^{4}\right]=E\left[E\left(u_{i}^{4} \mid X_{2 i}\right) f_{1 i}^{4} E\left(u_{j}^{4} \mid X_{2 j}\right) f_{1 j}^{4} K_{n i j}^{4}\right]=O\left(h^{-3 p_{2}}\right)
$$

As $G_{n}\left(Z_{i}, Z_{j}\right)=u_{i} f_{i} u_{j} f_{j} E\left[\sigma^{2}\left(X_{2,0}\right) f_{1}^{2}\left(X_{1,0}\right) K_{n i 0} K_{n j 0} \mid X_{2 i}, X_{2 j}\right]$, we have by Lemma 1

$$
\begin{aligned}
E & {\left[G_{n}^{2}\right] } \\
& =\int \sigma_{2 i}^{2} f_{i}^{2} \sigma_{2 j}^{2} f_{j}^{2}\left[\int \sigma_{2,0}^{2} f_{1}^{2}\left(X_{1,0}\right) K_{n i 0} K_{n j 0} f_{2}\left(X_{2,0}\right) d X_{2,0}\right]^{2} f_{2 i} f_{2 j} d X_{2 i} d X_{2 j} \\
& =h^{-2 p_{2}} \int \sigma_{2 i}^{2} f_{i}^{2} \sigma_{2 j}^{2} f_{j}^{2}\left[\int \sigma^{2}\left(X_{2 i}-h s\right) f_{1}^{2}\left(X_{1 i}-h s_{1}\right) K(s) K\left(s+\frac{X_{2 j}-X_{2 i}}{h}\right) f_{2}\left(X_{2 i}-h s\right) d s\right]^{2} f_{2 i} f_{2 j} d X_{2 i} d X_{2 j} \\
& =h^{-p_{2}} \int \sigma_{2 i}^{2} f_{i}^{2} \sigma^{2}\left(X_{2 i}+h t\right) f_{1}^{2}\left(X_{1 i}+h t_{1}\right) \\
& \quad\left[\int \sigma^{2}\left(X_{2 i}-h s\right) f_{1}^{2}\left(X_{1 i}-h s_{1}\right) K(s) K(s+t) f_{2}\left(X_{2 i}-h s\right) d s\right]^{2} f_{2 i} f_{2}\left(X_{2 i}+h t\right) d X_{2 i} d t \\
= & h^{-p_{2}} \int\left[\sigma^{2}\left(X_{2}\right)\right]^{4} f_{1}^{8}\left(X_{1}\right) f_{2}^{4}\left(X_{2}\right) d X_{2} \int\left[\int K(s) K(s+t) d s\right]^{2} d t+o\left(h^{-p_{2}}\right) \\
= & O\left(h^{-p_{2}}\right),
\end{aligned}
$$

where $s_{1}$ and $t_{1}$ denote the first $p_{1}$ elements of $s$ and $t$. Thus condition (4.1) holds as $h \rightarrow 0$ and $n h^{p_{2}} \rightarrow \infty$. Collecting results, Proposition 1 follows.

Q.E.D. 


\subsubsection{U-Statistics}

Let $U_{n}=\left(1 / n^{(m)}\right) \sum_{a} H_{n}\left(Z_{i_{1}}, \ldots, Z_{i_{m}}\right)$ be an arbitrary U-statistic, where the $Z_{i}$ 's are i.i.d. but $H_{n}$ is not necessarily symmetric. Then,

$$
\begin{aligned}
E\left(U_{n}^{2}\right) & =\left(\frac{1}{n^{(m)}}\right)^{2} \sum_{c=0}^{m} \frac{n^{(2 m-c)}}{c !} \sum_{\left|\Delta_{1}\right|=c=\left|\Delta_{2}\right|}^{(c)} I\left(\Delta_{1}, \Delta_{2}\right) \\
& =\sum_{c=0}^{m} O\left(n^{-c}\right) \sum_{\left|\Delta_{1}\right|=c=\left|\Delta_{2}\right|}^{(c)} I\left(\Delta_{1}, \Delta_{2}\right)
\end{aligned}
$$

where $\sum^{(c)}$ denotes summation over sets $\Delta_{1}$ and $\Delta_{2}$ of (ordered) positions of length $c$,

$$
I\left(\Delta_{1}, \Delta_{2}\right)=E\left[H_{n}\left(Z_{i_{1}}, \ldots, Z_{i_{m}}\right) H_{n}\left(Z_{j_{1}}, \ldots, Z_{j_{m}}\right)\right]
$$

and the $i$ 's in position $\Delta_{1}$ coincide with the $j$ 's in position $\Delta_{2}$ and are pairwise distinct otherwise. Note that this formula corrects equation (A.1) in Stute (1991). In what follows, we let $\xi_{c}=\sum^{(c)} I\left(\Delta_{1}, \Delta_{2}\right)$ and intensively use (4.2) to bound $E\left(U_{n}^{2}\right)$. Indeed, if $Z_{c}$ denotes the vector of common $Z_{i}$ 's, we have by conditioning on $Z_{c}$

$$
\begin{aligned}
I^{2}\left(\Delta_{1}, \Delta_{2}\right) & =E^{2}\left[E\left[H_{n}\left(Z_{i_{1}}, \ldots, Z_{i_{m}}\right) \mid Z_{c}\right] E\left[H_{n}\left(Z_{j_{1}}, \ldots, Z_{j_{m}}\right) \mid Z_{c}\right]\right] \\
& \leq E\left[E^{2}\left[H_{n}\left(Z_{i_{1}}, \ldots, Z_{i_{m}}\right) \mid Z_{c}\right]\right] E\left[E^{2}\left[H_{n}\left(Z_{j_{1}}, \ldots, Z_{j_{m}}\right) \mid Z_{c}\right]\right]
\end{aligned}
$$

by Cauchy-Schwartz inequality.

Proposition $2: n h^{p_{2} / 2} I_{1,3}=\delta_{n} \sqrt{n} h^{p_{2} / 2} O_{p}(1)+o_{p}(1)$.

Proof: $I_{1,3}$ is a U-statistic with kernel $H_{n}\left(Z_{i}, Z_{j}, Z_{l}\right)=u_{i} f_{i} u_{l} L_{n j l} K_{n i j}$. In order to use (4.2), we need to compute the corresponding $\xi_{c}, c=0,1,2,3$.

(i) $\xi_{2}=O\left(g^{-p_{1}}\right)$. Indeed we have

$$
\begin{aligned}
E\left(H_{n} \mid Z_{i}, Z_{j}\right) & =u_{i} f_{i} K_{n i j} E\left(u_{l} L_{n j l} \mid Z_{j}\right)=0, \\
E\left(H_{n} \mid Z_{i}, Z_{l}\right) & =u_{i} f_{i} u_{l} E\left(L_{n j l} K_{n i j} \mid Z_{i}, Z_{l}\right), \\
E\left(H_{n} \mid Z_{j}, Z_{l}\right) & =u_{l} L_{n j l} E\left(u_{i} f_{i} K_{n i j} \mid Z_{j}\right)=\delta_{n} u_{l} L_{n j l} E\left(d_{i} f_{i} K_{n i j} \mid Z_{j}\right) .
\end{aligned}
$$


Then, using $\boldsymbol{K} \equiv|K|$ and $\boldsymbol{L} \equiv|L|$, we have, by successive applications of Lemma 1,

$$
\begin{aligned}
E\left[E^{2}\left(H_{n} \mid Z_{i}, Z_{l}\right)\right] & =E\left[u_{i}^{2} f_{i}^{2} u_{l}^{2} E\left(L_{n j l} K_{n i j} \mid Z_{i}, Z_{l}\right) E\left(L_{n j^{\prime} l} K_{n i j^{\prime}} \mid Z_{i}, Z_{l}\right)\right] \\
& =O\left(g^{-p_{1}}\right) E\left[u_{i}^{2} f_{i}^{2} u_{l}^{2} E\left(\boldsymbol{L}_{n j l} \boldsymbol{K}_{n i j} \mid Z_{i}, Z_{l}\right) E\left(\boldsymbol{K}_{n i j^{\prime}} \mid Z_{i}, Z_{l}\right)\right] \\
& =O\left(g^{-p_{1}}\right) E\left[u_{i}^{2} f_{i}^{2} u_{l}^{2} \boldsymbol{L}_{n j l} \boldsymbol{K}_{n i j} f_{2}\left(X_{2 i}\right)\right]=O\left(g^{-p_{1}}\right), \\
E\left[E^{2}\left(H_{n} \mid Z_{j}, Z_{l}\right)\right] & =\delta_{n}^{2} E\left[u_{l}^{2} L_{n j l}^{2} E^{2}\left(d_{i} f_{i} K_{n i j} \mid Z_{j}\right)\right] \\
& =\delta_{n}^{2} E\left[u_{l}^{2} L_{n j l}^{2} d_{j}^{2} f_{j}^{2} f_{2}^{2}\left(X_{2 j}\right)\right] \\
& =\delta_{n}^{2} O\left(g^{-p_{1}}\right) E\left[u_{l}^{2} \boldsymbol{L}_{n j l} d_{j}^{2} f_{j}^{2} f_{2}^{2}\left(X_{2 j}\right)\right]=O\left(g^{-p_{1}}\right) .
\end{aligned}
$$

(ii) $\xi_{1}=O\left(\delta_{n}^{2}\right)$. Indeed, $E\left(H_{n} \mid Z_{i}\right)=E\left(H_{n} \mid Z_{j}\right)=0$ and $E\left(H_{n} \mid Z_{l}\right)=\delta_{n} u_{l} E\left(d_{i} f_{i} L_{n j l} K_{n i j} \mid Z_{l}\right)$. Then

$$
\begin{aligned}
E\left[E^{2}\left(H_{n} \mid Z_{l}\right)\right] & =\delta_{n}^{2} E\left[u_{l}^{2} E^{2}\left(d_{i} f_{i} L_{n j l} K_{n i j} \mid Z_{l}\right)\right] \\
& =O\left(\delta_{n}^{2}\right) E\left[u_{l}^{2} E^{2}\left(L_{n j l} d_{j} f_{j} f_{2 j} \mid Z_{l}\right)\right]=O\left(\delta_{n}^{2}\right) .
\end{aligned}
$$

(iii) $E\left[H_{n}\right]=0$. Thus $\xi_{0}=0$.

(iv) $\xi_{3}=O\left(g^{-p_{1}} h^{-p_{2}}\right)$, as $E\left[H_{n}^{2}\right]$ equals

$$
E\left[u_{i}^{2} u_{l}^{2} f_{i}^{2} L_{n j l}^{2} K_{n i j}^{2}\right]=O\left(g^{-p_{1}} h^{-p_{2}}\right) E\left[u_{i}^{2} u_{l}^{2} f_{i}^{2} \boldsymbol{L}_{n j l} \boldsymbol{K}_{n i j}\right]=O\left(g^{-p_{1}} h^{-p_{2}}\right) .
$$

Collecting results, $E\left(n h^{p_{2} / 2} I_{1,3}\right)^{2}=\delta_{n}^{2} n h^{p_{2}} O(1)+h^{p_{2}} / g^{p_{1}} O(1)+O\left(n g^{p_{1}}\right)^{-1}$.

Q.E.D.

Proposition $3: n h^{p_{2} / 2} I_{1,5}=o_{p}(1)$.

Proof: $I_{1,5}$ is a U-statistic with kernel $H_{n}\left(Z_{i}, Z_{j}, Z_{k}, Z_{l}\right)=u_{k} u_{l} L_{n i k} L_{n j l} K_{n i j}$.

(i) $\xi_{3}=O\left(g^{-2 p_{1}}\right)$. Indeed we have

$$
\begin{aligned}
E\left(H_{n} \mid Z_{i}, Z_{j}, Z_{k}\right) & =u_{k} L_{n i k} K_{n i j} E\left(u_{l} L_{n j l} \mid Z_{j}\right)=0, \\
E\left(H_{n} \mid Z_{i}, Z_{j}, Z_{l}\right) & =u_{l} L_{n j l} K_{n i j} E\left(u_{k} L_{n i k} \mid Z_{i}\right)=0, \\
E\left(H_{n} \mid Z_{i}, Z_{k}, Z_{l}\right) & =u_{k} u_{l} L_{n i k} E\left(L_{n j l} K_{n i j} \mid Z_{i}, Z_{l}\right), \\
E\left(H_{n} \mid Z_{j}, Z_{k}, Z_{l}\right) & =u_{k} u_{l} L_{n j l} E\left(L_{n i k} K_{n i j} \mid Z_{j}, Z_{k}\right) .
\end{aligned}
$$


Then, we have, by successive applications of Lemma 1

$$
\begin{aligned}
E\left[E^{2}\left(H_{n} \mid Z_{i}, Z_{k}, Z_{l}\right)\right] & =E\left[u_{k}^{2} u_{l}^{2} L_{n i k}^{2} E\left(L_{n j l} K_{n i j} \mid Z_{i}, Z_{l}\right) E\left(L_{n j^{\prime} l} K_{n i j^{\prime}} \mid Z_{i}, Z_{l}\right)\right] \\
& =O\left(g^{-2 p_{1}}\right) E\left[u_{k}^{2} u_{l}^{2} \boldsymbol{L}_{n i k} E\left(\boldsymbol{L}_{n j l} \boldsymbol{K}_{n i j} \mid Z_{i}, Z_{l}\right) E\left(\boldsymbol{K}_{n i j^{\prime}} \mid Z_{i}, Z_{l}\right)\right] \\
& =O\left(g^{-2 p_{1}}\right) E\left[u_{k}^{2} u_{l}^{2} \boldsymbol{L}_{n i k} \boldsymbol{L}_{n j l} \boldsymbol{K}_{n i j} f_{2 i}\right]=O\left(g^{-2 p_{1}}\right), \\
E\left[E^{2}\left(H_{n} \mid Z_{j}, Z_{k}, Z_{l}\right)\right] & =E\left[u_{k}^{2} u_{l}^{2} L_{n j l}^{2} E^{2}\left(L_{n i k} K_{n i j} \mid Z_{j}, Z_{k}\right)\right]=O\left(g^{-2 p_{1}}\right) .
\end{aligned}
$$

(ii) $\xi_{2}=O\left(g^{-p_{1}}\right)$. Indeed we have $E\left(H_{n} \mid Z_{i}, Z_{j}\right)=E\left(H_{n} \mid Z_{i}, Z_{k}\right)=E\left(H_{n} \mid Z_{i}, Z_{l}\right)=$ $E\left(H_{n} \mid Z_{j}, Z_{k}\right)=E\left(H_{n} \mid Z_{j}, Z_{l}\right)=0$ and $E\left(H_{n} \mid Z_{k}, Z_{l}\right)=u_{k} u_{l} E\left(L_{n i k} L_{n j l} K_{n i j} \mid Z_{k}, Z_{l}\right)$, and

$$
\begin{aligned}
E\left[E^{2}\left(H_{n} \mid Z_{k}, Z_{l}\right)\right] & =E\left[u_{k}^{2} u_{l}^{2} E\left(L_{n i k} L_{n j l} K_{n i j} \mid Z_{k}, Z_{l}\right) E\left(L_{n i^{\prime} k} L_{n j^{\prime} l} K_{n i j^{\prime}} \mid Z_{k}, Z_{l}\right)\right] \\
& =O\left(g^{-p_{1}}\right) E\left[u_{k}^{2} u_{l}^{2} E\left(\boldsymbol{L}_{n i k} \boldsymbol{L}_{n j l} \boldsymbol{K}_{n i j} \mid Z_{k}, Z_{l}\right) E\left(\boldsymbol{L}_{n j^{\prime} l} \boldsymbol{K}_{n i j^{\prime}} \mid Z_{k}, Z_{l}\right)\right] \\
& =O\left(g^{-p_{1}}\right) E\left[u_{k}^{2} u_{l}^{2} \boldsymbol{L}_{n i k} \boldsymbol{L}_{n j l} \boldsymbol{K}_{n i j} f_{l}^{2}\right]=O\left(g^{-p_{1}}\right) .
\end{aligned}
$$

(iii) $\xi_{1}=0$.

(iv) $E\left[H_{n}\right]=0$. Thus $\xi_{0}=0$.

(v) $\xi_{4}=O\left(g^{-2 p_{1}} h^{-p_{2}}\right)$, as $E\left[H_{n}^{2}\right]$ equals

$$
E\left[u_{k}^{2} u_{l}^{2} L_{n i k}^{2} L_{n j l}^{2} K_{n i j}^{2}\right]=O\left(g^{-2 p_{1}} h^{-p_{2}}\right) E\left[u_{k}^{2} u_{l}^{2} \boldsymbol{L}_{n i k} \boldsymbol{L}_{n j l} \boldsymbol{K}_{n i j}\right]=O\left(g^{-2 p_{1}} h^{-p_{2}}\right) .
$$

Collecting results $E\left(n h^{p_{2} / 2} I_{1,5}\right)^{2}=\left(h^{p_{2}} / g^{p_{1}}\right)\left[O(1)+O\left(n g^{p_{1}}\right)^{-1}\right]+O\left(n g^{p_{1}}\right)^{-2}$. Q.E.D.

Proposition $4: n h^{p_{2} / 2} I_{1,6}=\delta_{n}^{2} n h^{p_{2} / 2} o_{p}(1)+o_{p}(1)$

Proof: $(n-3) I_{1,6}=$ is a U-statistic with kernel $H_{n}\left(Z_{i}, Z_{j}, Z_{k}\right)=u_{i} u_{j} L_{n i k} L_{n j k} K_{n i j}$. Using a reasoning similar to the one followed in Proposition 3, it is not difficult to show that this U-statistic is such that $\xi_{3}=O\left(g^{-2 p_{1}} h^{-2 p_{2}}\right), \xi_{2}=O\left(g^{-2 p_{1}} h^{-p_{2}}\right)+O\left(g^{-p_{1}} h^{-2 p_{2}}\right)$, $\xi_{1}=O\left(h^{-2 p_{2}}\right)$ and $E\left[H_{n}\right]=O\left(\delta_{n}^{2} g^{-p_{1}}\right)$. Hence, $E\left(n h^{p_{2} / 2} I_{1,6}\right)^{2}=O\left(\delta_{n}^{2} n h^{p_{2} / 2}\right)^{2}\left(n g^{p_{1}}\right)^{-2}+$ $O\left(n h^{p_{2}}\right)^{-1}+O\left(n^{2} g^{p_{1}} h^{p_{2}}\right)^{-1}+O\left(n g^{p_{1}}\right)^{-2}+O\left(n g^{p_{1}}\right)^{-2}\left(n h^{p_{2}}\right)^{-1}$.

Q.E.D.

Proposition $5: n h^{p_{2} / 2} I_{2,1}=\delta_{n} \sqrt{n} h^{p_{2} / 2} o_{p}(1)+\delta_{n} n h^{p_{2} / 2} g^{\left(m_{1}+q_{1}\right)} O_{p}(1)+o_{p}(1)$.

Proof: $I_{2,1}=$ is a U-statistic with kernel $H_{n}\left(Z_{i}, Z_{j}, Z_{l}\right)=u_{i} f_{i}\left(r_{j}-r_{l}\right) L_{n j l} K_{n i j}$. 
(i) $\xi_{2}=o\left(h^{-p_{2}}\right)+o\left(g^{-p_{1}}\right)$. Indeed we have

$$
\begin{aligned}
E\left(H_{n} \mid Z_{i}, Z_{j}\right) & =u_{i} f_{i} K_{n i j} E\left(\left(r_{j}-r_{l}\right) L_{n j l} \mid Z_{j}\right), \\
E\left(H_{n} \mid Z_{i}, Z_{l}\right) & =u_{i} f_{i} E\left(\left(r_{j}-r_{l}\right) L_{n j l} K_{n i j} \mid Z_{i}, Z_{l}\right), \\
E\left(H_{n} \mid Z_{j}, Z_{l}\right) & =\delta_{n}\left(r_{j}-r_{l}\right) L_{n j l} E\left(d_{i} f_{i} K_{n i j} \mid Z_{j}\right) .
\end{aligned}
$$

First, we use the fact that $E\left[\left(r_{j}-r_{l}\right) L_{n j l} \mid Z_{j}\right]=O\left(g^{\left(m_{1}+q_{1}\right)}\right)=o(1)$ uniformly in $Z_{j}$ by a standard Taylor expansion argument, so that

$$
\begin{aligned}
E\left[E^{2}\left(H_{n} \mid Z_{i}, Z_{j}\right)\right] & =E\left[u_{i}^{2} f_{i}^{2} K_{n i j}^{2} E^{2}\left(\left(r_{j}-r_{l}\right) L_{n j l} \mid Z_{j}\right)\right] \\
& =O\left(h^{-p_{2}}\right) E\left[u_{i}^{2} f_{i}^{2} \boldsymbol{K}_{n i j} E^{2}\left(\left(r_{j}-r_{l}\right) L_{n j l} \mid Z_{j}\right)\right]=o\left(h^{-p_{2}}\right) .
\end{aligned}
$$

Now, by successive applications of Lemma 1,

$$
\begin{aligned}
E\left[E^{2}\left(H_{n} \mid Z_{i}, Z_{l}\right)\right] & =E\left[u_{i}^{2} f_{i}^{2} E\left(\left(r_{j}-r_{l}\right) L_{n j l} K_{n i j} \mid Z_{i}, Z_{l}\right) E\left(\left(r_{j^{\prime}}-r_{l}\right) L_{n j^{\prime} l} K_{n i j^{\prime}} \mid Z_{i}, Z_{l}\right)\right] \\
& =O\left(g^{-p_{1}}\right) E\left[u_{i}^{2} f_{i}^{2} E\left(\left|r_{j}-r_{l}\right| \boldsymbol{L}_{n j l} \boldsymbol{K}_{n i j} \mid Z_{i}, Z_{l}\right) E\left(\left|r_{j^{\prime}}-r_{l}\right| \boldsymbol{K}_{n i j^{\prime}} \mid Z_{i}, Z_{l}\right)\right] \\
& =o\left(g^{-p_{1}}\right), \\
E\left[E^{2}\left(H_{n} \mid Z_{j}, Z_{l}\right)\right] & =\delta_{n}^{2} E\left[\left(r_{j}-r_{l}\right)^{2} L_{n j l}^{2} E^{2}\left(d_{i} f_{i} K_{n i j} \mid Z_{j}\right)\right] \\
& =O\left(\delta_{n}^{2} g^{-p_{1}}\right) E\left[\left(r_{j}-r_{l}\right)^{2} \boldsymbol{L}_{n j l} d_{j}^{2} f_{j}^{2} f_{2 j}^{2}\right]=o\left(g^{-p_{1}}\right) .
\end{aligned}
$$

(ii) $\xi_{1}=O\left(g^{2\left(m_{1}+q_{1}\right)}\right)+o\left(\delta_{n}^{2}\right)$. Indeed, by a similar reasoning to (i),

$$
\begin{aligned}
E\left[E^{2}\left(H_{n} \mid Z_{i}\right)\right] & =E\left[u_{i}^{2} f_{i}^{2} E^{2}\left(K_{n i j} E\left(\left(r_{j}-r_{l}\right) L_{n j l} \mid Z_{j}\right) \mid Z_{i}\right)\right] \\
& =O\left(g^{2\left(m_{1}+q_{1}\right)}\right) E\left[u_{i}^{2} f_{i}^{2} E^{2}\left(\boldsymbol{K}_{n i j}\right)\right]=O\left(g^{2\left(m_{1}+q_{1}\right)}\right), \\
E\left[E^{2}\left(H_{n} \mid Z_{j}\right)\right] & =E\left[E^{2}\left(u_{i} f_{i}\left(r_{j}-r_{l}\right) L_{n j l} K_{n i j} \mid Z_{j}\right)\right]=O\left(g^{2\left(m_{1}+q_{1}\right)}\right), \\
E\left[E^{2}\left(H_{n} \mid Z_{l}\right)\right] & =E\left[E^{2}\left(\left(r_{j}-r_{l}\right) L_{n j l} E\left(u_{i} f_{i} K_{n i j} \mid Z_{j}\right) \mid Z_{l}\right)\right] \\
& =\delta_{n}^{2} E\left[E^{2}\left(\left(r_{j}-r_{l}\right) L_{n j l} d_{j} f_{j} f_{2 j} \mid Z_{l}\right)\right]=o\left(\delta_{n}^{2}\right) .
\end{aligned}
$$

(iii)

$$
\begin{aligned}
E\left(H_{n}\right) & =E\left[u_{i} f_{i}\left(r_{j}-r_{l}\right) L_{n j l} K_{n i j}\right] \\
& \approx \delta_{n} E\left[\left(r_{j}-r_{l}\right) L_{n j l} d_{j} f_{j} f_{2 j}\right] \\
& \approx O\left(\delta_{n} g^{\left(m_{1}+q_{1}\right)}\right) E\left[d_{j} f_{j} f_{2 j}\right]=O\left(\delta_{n} g^{\left(m_{1}+q_{1}\right)}\right) .
\end{aligned}
$$

(iv) $\xi_{3}=o\left(g^{-p_{1}} h^{-p_{2}}\right)$, as $E\left[H_{n}^{2}\right]=E\left[u_{i}^{2} f_{i}^{2}\left(r_{j}-r_{l}\right)^{2} L_{n j l}^{2} K_{n i j}^{2}\right]=o\left(g^{-p_{1}} h^{-p_{2}}\right)$. 
Collecting results, $E\left(n h^{p_{2} / 2} I_{2,1}\right)^{2}=\delta_{n}^{2} n^{2} h^{p_{2}} O\left(g^{2\left(m_{1}+q_{1}\right)}\right)+n h^{p_{2}} O\left(g^{2\left(m_{1}+q_{1}\right)}\right)+o\left(\delta_{n}^{2} n h^{p_{2}}\right)+$ $o(1)+o\left(h^{p_{2}} / g^{p_{1}}\right)+o\left(n g^{p_{1}}\right)^{-1}$.

Q.E.D.

Proposition $6: n h^{p_{2} / 2} I_{2,3}=o_{p}(1)$.

Proof: $I_{2,3}$ is a U-statistic with kernel $H_{n}\left(Z_{i}, Z_{j}, Z_{k}, Z_{l}\right)=u_{k}\left(r_{j}-r_{l}\right) L_{n i k} L_{n j l} K_{n i j}$. Using a reasoning similar to the one followed in Proposition (5), it is not difficult to show that this U-statistic is such that $\xi_{4}=o\left(h^{-p_{2}} g^{-2 p_{1}}\right), \xi_{3}=o\left(g^{-p_{1}} h^{-p_{2}}\right)+o\left(g^{-2 p_{1}}\right), \xi_{2}=$ $o\left(g^{-p_{1}}\right), \xi_{1}=O\left(g^{2\left(m_{1}+q_{1}\right)}\right)$ and $E\left[H_{n}\right]=0$. Hence, $E\left(n h^{p_{2} / 2} I_{2,3}\right)^{2}=n h^{p_{2}} O\left(g^{2\left(m_{1}+q_{1}\right)}\right)+$ $o\left(h^{p_{2}} / g^{p_{1}}\right)\left[1+\left(n g^{p_{1}}\right)^{-1}\right]+o\left(n g^{p_{1}}\right)^{-1}+o\left(n g^{p_{1}}\right)^{-2}$.

Q.E.D.

Proposition $7: n h^{p_{2} / 2} I_{3}=n h^{p_{2} / 2} O_{p}\left(g^{2\left(m_{1}+q_{1}\right)}\right)+o_{p}(1)$.

Proof: $I_{3}$ is a U-statistic with kernel $H_{n}\left(Z_{i}, Z_{j}, Z_{k}, Z_{l}\right)=\left(r_{i}-r_{k}\right)\left(r_{j}-r_{l}\right) L_{n i k} L_{n j l} K_{n i j}$. Similarly to the proof of Proposition 5 for $I_{2,3}$, we can show that $\xi_{4}=o\left(h^{-p_{2}} g^{-2 p_{1}}\right)$, $\xi_{3}=o\left(h^{-p_{2}} g^{-p_{1}}\right)+o\left(g^{-2 p_{1}}\right), \xi_{2}=o\left(g^{-p_{1}}\right), \xi_{1}=o\left(g^{2\left(m_{1}+q_{1}\right)}\right)$. On the other hand,

$$
E\left[H_{n}\right]=E\left[\left(r_{i}-r_{k}\right)\left(r_{j}-r_{l}\right) L_{n i k} L_{n j l} K_{n i j}\right]=O\left(g^{2\left(m_{1}+q_{1}\right)}\right),
$$

so that $E\left(n h^{p_{2} / 2} I_{3}\right)^{2}=n^{2} h^{p_{2}} O\left(g^{4\left(m_{1}+q_{1}\right)}\right)+o(1)$.

\subsubsection{The remaining terms}

Proposition $8: n h^{p_{2} / 2} I_{1,1}=\delta_{n}^{2} n h^{p_{2} / 2} o_{p}(1)+\delta_{n} \sqrt{n} h^{p_{2} / 2} o_{p}(1)+o_{p}(1)$.

Proof: We denote $\left(\hat{f}_{i}^{j}-f_{i}\right)$ by $\Delta f_{i}^{j}$. We have $I_{1,1}=\left(1 / n^{(2)}\right) \sum_{a} u_{i} \Delta f_{i}^{j} u_{j} f_{j} K_{n i j}$ so that

$$
E\left(I_{1,1}^{2}\right)=\left(\frac{1}{n^{(2)}}\right)^{2}\left[\sum_{a} u_{i} \Delta f_{i}^{j} u_{j} f_{j} K_{n i j}\right]\left[\sum_{a} u_{i^{\prime}} \Delta f_{i^{\prime}}^{j^{\prime}} u_{j^{\prime}} f_{j^{\prime}} K_{n i^{\prime} j^{\prime}}\right],
$$

where the first (respectively the second) sum is taken over all arrangements of different indices $i$ and $j$ (respectively different indices $i^{\prime}$ and $j^{\prime}$ ).

Let $\bar{X}_{1}$ be the $\sigma$-algebra generated by all the $X_{1 i}$ 's and $\lambda_{n}$ be $E\left[\Delta^{2} f_{i}^{j} \mid Z_{i}, Z_{j}, Z_{i^{\prime}}, Z_{j^{\prime}}\right]$, which is $o(1)$ uniformly by Lemma 2 . We consider three situations. 
(i) All indices are different: $n^{(4)}$ terms.

$$
\begin{aligned}
E\left[u_{i} \Delta f_{i}^{j} u_{j} f_{j} K_{n i j} u_{i^{\prime}} \Delta f_{i^{\prime}}^{j^{\prime}} u_{j^{\prime}} f_{j^{\prime}} K_{n i^{\prime} j^{\prime}}\right] & =\delta_{n}^{4} E\left[\Delta f_{i}^{j} f_{j} \Delta f_{i^{\prime}}^{j^{\prime}} f_{j^{\prime}} E\left(d_{i} d_{j} d_{i^{\prime}} d_{j^{\prime}} K_{n i j} K_{n i^{\prime} j^{\prime}} \mid \bar{X}_{1}\right)\right] \\
& =\delta_{n}^{4} E\left[f_{j} f_{j^{\prime}} d_{i} d_{j} d_{i^{\prime}} d_{j^{\prime}} K_{n i j} K_{n i^{\prime} j^{\prime}} E\left(\Delta f_{i}^{j} \Delta f_{i^{\prime}}^{j^{\prime}} \mid Z_{i}, Z_{j}, Z_{i^{\prime}}, Z_{j^{\prime}}\right)\right] \\
& =\delta_{n}^{4} \lambda_{n} E\left|f_{j} f_{j^{\prime}} d_{i} d_{j} d_{i^{\prime}} d_{j^{\prime}} K_{n i j} K_{n i^{\prime} j^{\prime}}\right|=O\left(\delta_{n}^{4} \lambda_{n}\right) .
\end{aligned}
$$

(ii) One index is common to $\{i, j\}$ and $\left\{i^{\prime}, j^{\prime}\right\}: 4 n^{(3)}$ terms.

$$
\begin{aligned}
&\left(i^{\prime}=i\right) E\left[u_{i}^{2} \Delta f_{i}^{j} u_{j} f_{j} K_{n i j} \Delta f_{i}^{j^{\prime}} u_{j^{\prime}} f_{j^{\prime}} K_{n i j^{\prime}}\right]=\delta_{n}^{2} E\left[\Delta f_{i}^{j} f_{j} \Delta f_{i}^{j^{\prime}} f_{j^{\prime}} E\left(u_{i}^{2} d_{j} d_{j^{\prime}} K_{n i j} K_{n i j^{\prime}} \mid \bar{X}_{1}\right)\right] \\
&=\delta_{n}^{2} \lambda_{n} E\left|f_{j} f_{j^{\prime}} u_{i}^{2} d_{j} d_{j^{\prime}} K_{n i j} K_{n i j^{\prime}}\right|=O\left(\delta_{n}^{2} \lambda_{n}\right), \\
&\left(\begin{array}{rl}
\left.j^{\prime}=j\right) \quad E\left[u_{i} \Delta f_{i}^{j} u_{j}^{2} f_{j}^{2} K_{n i j} u_{i^{\prime}} \Delta f_{i^{\prime}}^{j} K_{n i^{\prime} j}\right] & =\delta_{n}^{2} E\left[\Delta f_{i}^{j} f_{j}^{2} \Delta f_{i^{\prime}}^{j} E\left(d_{i} u_{j}^{2} d_{i^{\prime}} K_{n i j} K_{n i^{\prime} j} \mid \bar{X}_{1}\right)\right] \\
& =\delta_{n}^{2} \lambda_{n} E\left|f_{j}^{2} d_{i} u_{j}^{2} d_{i^{\prime}} K_{n i j} K_{n i^{\prime} j}\right|=O\left(\delta_{n}^{2} \lambda_{n}\right),
\end{array}\right. \\
&\left(\begin{array}{rl}
\left(i^{\prime}=j\right) \quad E\left[u_{i} \Delta f_{i}^{j} u_{j}^{2} f_{j} K_{n i j} \Delta f_{j}^{j^{\prime}} u_{j^{\prime}} f_{j^{\prime}} K_{n j j^{\prime}}\right] & =\delta_{n}^{2} E\left[\Delta f_{i}^{j} f_{j} \Delta f_{j}^{j^{\prime}} f_{j^{\prime}} E\left(d_{i} u_{j}^{2} d_{j^{\prime}} K_{n i j} K_{n j j^{\prime}} \mid \bar{X}_{1}\right)\right] \\
& =\delta_{n}^{2} \lambda_{n} E\left|f_{j} f_{j^{\prime}} d_{i} u_{j}^{2} d_{j^{\prime}} K_{n i j} K_{n j j^{\prime}}\right|=O\left(\delta_{n}^{2} \lambda_{n}\right) .
\end{array}\right.
\end{aligned}
$$

The case $j^{\prime}=i$ is similar to $i^{\prime}=j$.

(iii) Two indices in common to $\{i, j\}$ and $\left\{i^{\prime}, j^{\prime}\right\}: 2 n^{(2)}$ terms. We have

$$
E\left[u_{i}^{2} u_{j}^{2}\left(\Delta f_{i}^{j}\right)^{2} f_{j}^{2} K_{n i j}^{2}\right]=O\left(\lambda_{n} / h^{p_{2}}\right) \text { and } E\left[u_{i}^{2} u_{j}^{2} \Delta f_{i}^{j} \Delta f_{j}^{i} f_{i} f_{j} K_{n i j}^{2}\right]=O\left(\lambda_{n} / h^{p_{2}}\right) .
$$

Therefore,

$$
E\left(n h^{p_{2} / 2} I_{1,1}\right)^{2}=\delta_{n}^{4} n^{2} h^{p_{2}} O\left(\lambda_{n}\right)+\delta_{n}^{2} n h^{p_{2}} O\left(\lambda_{n}\right)+O\left(\lambda_{n}\right) .
$$

The proposition then follows from $\lambda_{n}=o(1)$ uniformly, see Lemma 2.

Q.E.D.

Proposition $9: n h^{p_{2} / 2} I_{1,2}=\delta_{n}^{2} n h^{p_{2} / 2} o_{p}(1)+\delta_{n} \sqrt{n} h^{p_{2} / 2} o_{p}(1)+o_{p}(1)$.

Proof: The proof is very similar to the proof of Proposition 8 for $I_{1,1}$ and is not reported.

Proposition $10: n h^{p_{2} / 2} I_{1,4}=\delta_{n}^{2} n h^{p_{2} / 2} o_{p}(1)+\delta_{n} \sqrt{n} h^{p_{2} / 2} o_{p}(1)+o_{p}(1)$

Proof: We denote $\left(\hat{f}_{i}^{j, l}-f_{i}\right)$ by $\Delta f_{i}^{j, l}$. We have $I_{1,4}=\left(1 / n^{(3)}\right) \sum_{a} u_{i} \Delta f_{i}^{j, l} u_{l} L_{n j l} K_{n i j}$ and

$$
E\left(I_{1,4}^{2}\right)=\left(\frac{1}{n^{(3)}}\right)^{2}\left[\sum_{a} u_{i} \Delta f_{i}^{j, l} u_{l} L_{n j l} K_{n i j}\right]\left[\sum_{a} u_{i^{\prime}} \Delta f_{i^{\prime}}^{j^{\prime}, l^{\prime}} u_{l^{\prime}} L_{n j^{\prime} l^{\prime}} K_{n i^{\prime} j^{\prime}}\right],
$$

where the first (respectively the second) sum is taken over all arrangements of pairwise different indices $i, j$ and $l$ (respectively pairwise different indices $i^{\prime}, j^{\prime}$ and $l^{\prime}$ ). Let $\bar{X}_{1}$ be 
the $\sigma$-algebra generated by all the $X_{1 i}$ 's and where $\lambda_{n}$ be $E\left[\Delta^{2} f_{i}^{j, l} \mid Z_{i}, Z_{j}, Z_{l}, Z_{i^{\prime}}, Z_{j^{\prime}}, Z_{l^{\prime}}\right]$, which is $o(1)$ uniformly by Lemma 2 . We consider four situations and employ a similar strategy as in Proposition 8's proof.

(i) All indices are different: $n^{(6)}$ terms.

$$
\begin{aligned}
& E\left[u_{i} \Delta f_{i}^{j, l} u_{l} L_{n j l} K_{n i j} u_{i^{\prime}} \Delta f_{i^{\prime}}^{j^{\prime}, l^{\prime}} u_{l^{\prime}} L_{n, j^{\prime} l^{\prime}} K_{n i^{\prime} j^{\prime}}\right] \\
& =\delta_{n}^{4} E\left[L_{n j l} L_{n j^{\prime} l^{\prime}} d_{i} d_{l} d_{i^{\prime}} d_{l^{\prime}} K_{n i j} K_{n i^{\prime} j^{\prime}} E\left(\Delta f_{i}^{j, l} \Delta f_{i^{\prime}}^{j^{\prime}, l^{\prime}} \mid Z_{i}, Z_{j}, Z_{l}, Z_{i^{\prime}}, Z_{j^{\prime}}, Z_{l^{\prime}}\right)\right] \\
& =O\left(\delta_{n}^{4} \lambda_{n}\right) .
\end{aligned}
$$

(ii) One index is common to $\{i, j, l\}$ and $\left\{i^{\prime}, j^{\prime}, l^{\prime}\right\}: 9 n^{(5)}$ terms. For case $\left(i^{\prime}=i\right)$, $E\left[u_{i}^{2} \Delta f_{i}^{j, l} u_{l} L_{n j l} K_{n i j} \Delta f_{i}^{j^{\prime}, l^{\prime}} u_{l^{\prime}} L_{n j^{\prime} l^{\prime}} K_{n i j^{\prime}}\right]=\delta_{n}^{2} E\left[\Delta f_{i}^{j, l} L_{n j l} \Delta f_{i}^{j^{\prime}, l^{\prime}} L_{n j^{\prime} l^{\prime}} u_{i}^{2} d_{l} d_{l^{\prime}} K_{n i j} K_{n i j^{\prime}}\right]=$ $O\left(\delta_{n}^{2} \lambda_{n}\right)$. Similar computations for the other cases lead to the same result.

(iii) Two indices are common to $\{i, j, l\}$ and $\left\{i^{\prime}, j^{\prime}, l^{\prime}\right\}: 18 n^{(4)}$ terms.

$$
\begin{aligned}
& \left(i=i^{\prime} \text { and } j=j^{\prime}\right) \\
& \begin{aligned}
E\left[u_{i}^{2} \Delta f_{i}^{j, l} u_{l} L_{n j l} \Delta f_{i}^{j, l^{\prime}} u_{l^{\prime}} L_{n j l^{\prime}} K_{n i j}^{2}\right] & =\delta_{n}^{2} E\left[\Delta f_{i}^{j, l} L_{n j l} \Delta f_{i}^{j, l^{\prime}} L_{n j l^{\prime}} u_{i}^{2} d_{l} d_{l^{\prime}} K_{n i j}^{2}\right] \\
& =O\left(\delta_{n}^{2} \lambda_{n} / h^{p_{2}}\right)=O\left(\lambda_{n} / h^{p_{2}}\right),
\end{aligned} \\
& \left(i=i^{\prime} \text { and } l=l^{\prime}\right) \\
& E\left[u_{i}^{2} \Delta f_{i}^{j, l} u_{l}^{2} L_{n j l} K_{n i j} \Delta f_{i}^{j^{\prime}, l} L_{n j^{\prime} l} K_{n i j^{\prime}}\right]
\end{aligned}
$$

In other cases, similar computations lead to either $O\left(\lambda_{n} / g^{p_{1}}\right)$ or $O\left(\lambda_{n} / h^{p_{2}}\right)$.

(iv) Three indices are common to $\{i, j, l\}$ and $\left\{i^{\prime}, j^{\prime}, l^{\prime}\right\}: 6 n^{(3)}$ terms. For instance, if $\left(i=i^{\prime}, j=j^{\prime}\right.$ and $\left.l=l^{\prime}\right), E\left[u_{i}^{2}\left(\Delta f_{i}^{j, l}\right)^{2} u_{l}^{2} L_{n j l}^{2} K_{n i j}^{2}\right]=O\left(\lambda_{n} /\left(g^{p_{1}} h^{p_{2}}\right)\right)$. In the remaining cases, the corresponding expectations are all $O\left(\lambda_{n} /\left(g^{p_{1}} h^{p_{2}}\right)\right)$.

Therefore from (4.2) we get

$E\left[n h^{p_{2} / 2} I_{1,4}\right]^{2}=\delta_{n}^{4} n^{2} h^{p_{2}} O\left(\lambda_{n}\right)+\delta_{n}^{2} n h^{p_{2}} O\left(\lambda_{n}\right)+h^{p_{2}} / g^{p_{1}} O\left(\lambda_{n}\right)+O\left(\lambda_{n}\right)+\left(n g^{p_{1}}\right)^{-1} O\left(\lambda_{n}\right)$.

The proposition then follows from $\lambda_{n}=o(1)$ uniformly, see Lemma 2 .

Q.E.D.

Proposition $11: n h^{p_{2} / 2} I_{2,2}=\delta_{n}^{2} n h^{p_{2} / 2} o_{p}(1)+\delta_{n} \sqrt{n} h^{p_{2} / 2} o_{p}(1)+o_{p}(1)$

Proof: The proof is very similar to the proof of Proposition 10 for $I_{1,4}$ and is not reported. 


\subsubsection{Lemmas}

Lemma 1 For any function $l(\cdot) \in \mathcal{U}^{p}$ and any integrable kernel $K(\cdot)$,

$$
\sup _{x \in \mathbb{R}^{p}}\left|\int l(X) \frac{1}{h^{p}} K\left(\frac{x-X}{h}\right) d X-l(x) \int K(u) d u\right| \rightarrow 0 .
$$

Proof: This result comes from the well-known Bochner lemma.

Lemma 2 : If the density $f_{1}\left(X_{1}\right) \in \mathcal{U}^{p_{1}}$ and $n g^{p_{1}} \rightarrow \infty, E\left[\Delta^{2} f_{i}^{j} \mid Z_{i}, Z_{j}, Z_{i^{\prime}}, Z_{j^{\prime}}\right]=o(1)$ and $E\left[\Delta^{2} f_{i}^{j, l} \mid Z_{i}, Z_{j}, Z_{l}, Z_{i^{\prime}}, Z_{j^{\prime}}, Z_{l^{\prime}}\right]=o(1)$ uniformly in the indices.

Proof: From the definition of $\Delta f_{i}^{j}$,

$$
\begin{aligned}
E\left[\Delta^{2} f_{i}^{j} \mid Z_{i}, Z_{j}, Z_{i^{\prime}}, Z_{j^{\prime}}\right]= & E\left[\left(\hat{f}_{i}^{j}-E\left(\hat{f}_{i}^{j} \mid Z_{i}, Z_{j}, Z_{i^{\prime}}, Z_{j^{\prime}}\right)\right)^{2} \mid Z_{i}, Z_{j}, Z_{i^{\prime}}, Z_{j^{\prime}}\right] \\
& \left.+\left[E\left(\hat{f}_{i}^{j} \mid Z_{i}, Z_{j}, Z_{i^{\prime}}, Z_{j^{\prime}}\right)\right)-f_{i}\right]^{2}
\end{aligned}
$$

Because $\hat{f}_{i}^{j}-E\left(\hat{f}_{i}^{j} \mid Z_{i}, Z_{j}, Z_{i^{\prime}}, Z_{j^{\prime}}\right)=(n-2)^{-1} \sum_{k \notin\left\{i, j, i^{\prime}, j^{\prime}\right\}}\left(L_{n i k}-E\left(L_{n i k} \mid Z_{i}\right)\right)$, whose summands are, conditional on $Z_{i}$, independent with zero mean,

$E\left[\left(\hat{f}_{i}^{j}-E\left(\hat{f}_{i}^{j} \mid Z_{i}, Z_{j}, Z_{i^{\prime}}, Z_{j^{\prime}}\right)\right)^{2} \mid Z_{i}, Z_{j}, Z_{i^{\prime}}, Z_{j^{\prime}}\right] \leq(n-2)^{-2} \sum_{k \notin\left\{i, j, i^{\prime}, j^{\prime}\right\}} E\left(L_{n i k}^{2} \mid Z_{i}\right)=O\left(n^{-1} g^{-p_{1}}\right)$.

As $E\left(\hat{f}_{i}^{j} \mid Z_{i}, Z_{j}, Z_{i^{\prime}}, Z_{j^{\prime}}\right)=(n-2)^{-1}\left[L_{n i i^{\prime}}+L_{n i j^{\prime}}+(n-4) E\left(L_{n i k} \mid Z_{i}\right)\right]$,

$$
\begin{aligned}
{\left[E\left(\hat{f}_{i}^{j} \mid Z_{i}, Z_{j}, Z_{i^{\prime}}, Z_{j^{\prime}}\right)-f_{i}\right]^{2} } & =\left[\frac{1}{n-2}\left(L_{n i i^{\prime}}+L_{n i j^{\prime}}-f_{i}\right)+\frac{n-4}{n-2} E\left(L_{n i k}-f_{i} \mid Z_{i}\right)\right]^{2} \\
& \leq\left[O\left(n^{-1} g^{-p_{1}}\right)+O\left(n^{-1}\right)+o(1)\right]^{2}=o(1) .
\end{aligned}
$$

The proof for the second part is similar and is therefore not reported.

Q.E.D.

\subsection{Proof of Corollary 1}

Let $u_{i}=Y_{i}-c$. As $Y_{i}-Y_{k}=\left(u_{i}-u_{k}\right)$, and as $K(\cdot)$ is even, we have

$$
V_{n}^{*}=\frac{1}{n^{(2)}} \sum_{a} u_{i} u_{j} K_{n i j}-\frac{2}{n^{(3)}} \sum_{a} u_{i} u_{l} K_{n i j}+\frac{1}{n^{(4)}} \sum_{a} u_{k} u_{l} K_{n i j}=V_{0 n}^{*}-2 V_{1 n}^{*}+V_{2 n}^{*},
$$


Each of these terms can be studied using the same techniques as in Theorem 1's proof, so that we have

$$
n h^{p_{2} / 2} V_{0 n}^{*}=A_{n}^{*}+\delta_{n}^{2} n h^{p_{2} / 2} \mu_{n}^{*}+\delta_{n} \sqrt{n} h^{p_{2} / 2} B_{n}^{*},
$$

where $A_{n}^{*} \stackrel{d}{\longrightarrow} N\left(0, \omega^{* 2}\right), \mu_{n}^{*} \longrightarrow \mu^{*}, B_{n}^{*} \stackrel{d}{\longrightarrow} 2 N\left(0, \xi^{*}-\delta^{2} \mu^{* 2}\right)$, with $\delta=\lim _{n \rightarrow \infty} \delta_{n}$ and $\xi^{*}=E\left[(Y-c)^{2} d^{2}\left(X_{2}\right) f^{2}\left(X_{2}\right)\right]$,

$$
n h^{p_{2} / 2} V_{1 n}^{*}=\delta_{n} \sqrt{n} h^{p_{2} / 2} O_{p}(1)+o_{p}(1) \text { and } n h^{p_{2} / 2} V_{2 n}^{*}=o_{p}(1)
$$

Collecting results, it follows that

$$
n h^{p_{2} / 2} V_{n}^{*}=A_{n}^{*}+\delta_{n}^{2} n h^{p_{2} / 2} \mu_{n}^{*}+\delta_{n} \sqrt{n} h^{p_{2} / 2} O_{p}(1)+o_{p}(1),
$$

where $A_{n}^{*} \stackrel{d}{\longrightarrow} N\left(0, \omega^{* 2}\right)$ and $\mu_{n}^{*} \rightarrow \mu^{*}$. The end of the proof is similar to Theorem 1's one. Q.E.D.

\section{REFERENCES}

AÏT-SAHALIA, Y., P. BICKEL and T. M. STOKER (1994): "Goodness-of-fit tests for regression using kernel methods," University of Chicago.

BIERENS, H.J. (1982): “Consistent model specification tests," Journal of Econometrics, 20, pp. 105-134.

BIERENS, H.J. (1990): “A consistent conditional moment test of functional form," Econometrica, 58, pp. $1443-1458$.

BUCKLEY, M.J. (1991): “Detecting a smooth signal: optimality of cusum based proceudres," Biometrika, 78 , pp. $253-262$.

BUCKLEY, M.J. and G.K. EAGLESON (1988): "An approximation to the distribution of quadratic forms in normal random variables," Australian Journal of Statistics, 30A, pp. 149-163.

CHEN, J.C. (1994): "Testing goodness-of-fit of polynomial models vai spline smoothing techniques," Statistics and Probability Letters, 19, pp. 65-76.

CLEVELAND, W.S. and S.J. DEVLIN (1988): "Locally weighted regression: an approach to regression analysis by local fitting," Journal of the American Statistical Association, 83 (403), pp. 596-610.

DIEBOLT, J. (1995): “A nonparametric test for the regression function: asymptotic theory," Journal of Statistical Planning and Inference, 44, pp. 1-17. 
EPANECHNIKOV, V. (1969): "Nonparametric estimates of a multivariate probability density," Theory of Probability and its Applications, 14, pp. 153-158.

EUBANK, R.L. and C.H. SPIEGELMAN (1990) : "Testing the goodness of fit of a linear model via nonparametric regression techniques," Journal of the American Statistical Association, 85 (410), pp. $387-392$.

EUBANK, R.L. and J.D. HART (1992): “Testing goodness-of-fit in regression via order selection criteria," Annals of Statistics, 20(3), pp. 1412-1425.

EUBANK, R.L. and J.D. HART (1993): "Commonality of cusum, von Neumann and smoothing-based goodness-of-fit tests," Biometrika, 80, pp. 89-98.

EUBANK, R.L. and V.N. LARICCIA (1993): "Testing for no effect in nonparametric regression," Journal of Statistical Planning and Inference, 36, pp. 1-14.

FAN, Y. and Q. LI (1996): “Consistent model specification tests: omitted variables and semiparametric functional forms," Econometrica, 64 (4), pp. 865-890.

GOZALO, P.L. (1993) : "A consistent model specification test for nonparametric estimation of regression functions models," Econometric Theory, 9, pp. 451-477.

GOZALO, P.L. (1995): “Nonparametric specification testing with $\sqrt{n}$ - local power and bootstrap critical values," Brown University.

GUERRE, E. and P. LAVERGNE (1998): "Minimax nonparametric tests for specification of regression models," Université Paris VI.

HALL, P. (1983): "Chi-squared approximations to the distribution of a sum of independent random variables," Annals of Probability, 11, pp. 1028-1036.

HALL, P. (1984a) : “Central limit theorem for integrated square error of multivariate nonparametric density estimators," Journal of Multivariate Analysis, 14, pp. 1-16.

HALL, P. (1984b) : “Integrated square error properties of kernel estimators of regression functions," Annals of Statistics, 12 (1), pp. 163-179.

HÄRDLE, W. (1991): Smoothing techniques with implementation in S. New-York: Springer Verlag.

HÄRDLE, W. and E. MAMMEN (1993): "Comparing nonparametric versus parametric regression fits," Annals of Statistics, 21 (4), pp. 1926-1947.

HART, J.D. (1997): Nonparametric smoothing and lack-of-fit tests. New-York: Springer Verlag.

HEFFERNAN, P.M. (1997): “Unbiased estimation of central moments by using U-statistics," Journal of the Royal Statistical Society, Series B, 59(4), pp. 861-863. 
HOEFFDING, W. (1948): “A class of statistics with asymptotically normal distribution," Annals of Mathematical Statistics, 15, pp. 293-325.

HONG, Y. (1993) : "Consistent specification testing using optimal nonparametric kernel estimation," Cornell University.

HONG, Y. and H. WHITE (1995): "Consistent specification testing via nonparametric series regression," Econometrica, 63 (5), pp. 1133-1159.

HOROWITZ, J.L. and W. HÄRDLE (1994): "Testing a parametric model against a semiparametric alternative," Econometric Theory, 10, pp. 821-848.

KUCHIBHATLA, M. and J.D. HART (1996): "Smoothing-based lack-of-fit tests: variations on a theme," Journal of Nonparametric Statistics, 7, pp. 1-22.

LAVERGNE, P. (1997): “An equality test across nonparametric regressions,” INRA-ESR.

LAVERGNE, P. and Q.H. VUONG (1996) : "Nonparametric selection of regressors: the nonnested case," Econometrica, 64 (1), 207-219.

ROBINSON, P.M. (1983): “Nonparametric estimators for time series," Journal of Time Series Analysis, 4, pp. $185-207$.

SAMAROV, A.M. (1993) : "Exploring regression structure using nonparametric functional estimation," Journal of the American Statistical Association, 88 (423), pp. 836-847.

STUTE, W. (1991): “Conditional U-statistics," Annals of Probability, 19 (2), pp. 812-825.

STUTE, W. (1997): “Nonparametric model checks for regression," Annals of Statistics, 25, pp. 613-641.

YATCHEW, A.J. (1992) : "Nonparametric regression tests based on least squares," Econometric Theory, 8, pp. $435-451$.

ZHENG, J.X. (1996): "A consistent test of functional form via nonparametric estimation techniques," Journal of Econometrics, 75 (2) pp. 263-289. 
Table 1: Null and Linear Alternatives

\begin{tabular}{|c|c|c|c|c|c|c|c|c|c|}
\hline \multirow{2}{*}{$\frac{n}{100}$} & \multirow{2}{*}{$\frac{c_{2}}{0.25}$} & \multicolumn{2}{|c|}{ DGP0 } & \multicolumn{2}{|c|}{ DGP1 } & \multicolumn{2}{|c|}{ DGP2 } & \multicolumn{2}{|c|}{ DGP3 } \\
\hline & & -0.019 & $(0.876)$ & 0.148 & $(0.909)$ & 0.583 & $(0.949)$ & 1.683 & $(0.894)$ \\
\hline & & -0.350 & $(0.959)$ & -0.159 & $(0.998)$ & 0.340 & (1.044) & 1.620 & $(0.973)$ \\
\hline & & $3.3 \%$ & $8.7 \%$ & $5.6 \%$ & $11.8 \%$ & $14.3 \%$ & $25.3 \%$ & $53.7 \%$ & $69.4 \%$ \\
\hline & & $2.1 \%$ & $5.2 \%$ & $3.7 \%$ & $8.5 \%$ & $11.5 \%$ & $20.0 \%$ & $51.3 \%$ & $66.1 \%$ \\
\hline & 0.5 & -0.007 & $(0.857)$ & 0.231 & $\overline{(0.922)}$ & 0.838 & $\overline{(1.009)}$ & 2.353 & $(0.986)$ \\
\hline & & -0.462 & $(0.941)$ & -0.190 & (1.014) & 0.506 & (1.112) & 2.268 & (1.075) \\
\hline & & $4.1 \%$ & $8.5 \%$ & $7.8 \%$ & $14.1 \%$ & $21.5 \%$ & $33.4 \%$ & $77.0 \%$ & $85.8 \%$ \\
\hline & & $2.0 \%$ & $4.8 \%$ & $5.1 \%$ & $8.7 \%$ & $16.1 \%$ & $24.4 \%$ & $73.1 \%$ & $82.3 \%$ \\
\hline & 1.0 & 0.010 & $(0.800)$ & 0.335 & $(0.918)$ & 1.170 & $(1.085)$ & 3.241 & $(1.136)$ \\
\hline & & -0.620 & $(0.877)$ & -0.247 & $(1.010)$ & 0.711 & (1.197) & 3.120 & (1.238) \\
\hline & & $3.6 \%$ & $7.4 \%$ & $9.0 \%$ & $15.4 \%$ & $31.3 \%$ & $43.4 \%$ & $91.9 \%$ & $96.1 \%$ \\
\hline & & $1.5 \%$ & $3.3 \%$ & $4.8 \%$ & $8.4 \%$ & $21.8 \%$ & $30.3 \%$ & $88.6 \%$ & $92.9 \%$ \\
\hline & 2.0 & 0.018 & $(0.707)$ & 0.450 & $(0.895)$ & 1.556 & $(1.173)$ & 4.279 & $(1.329)$ \\
\hline & & -0.852 & $(0.779)$ & -0.358 & $(0.989)$ & 0.909 & (1.299) & 4.070 & $(1.450)$ \\
\hline & & $2.4 \%$ & $5.3 \%$ & $9.8 \%$ & $16.9 \%$ & $42.6 \%$ & $54.3 \%$ & $98.0 \%$ & $99.0 \%$ \\
\hline & & $0.7 \%$ & $1.6 \%$ & $4.1 \%$ & $6.4 \%$ & $27.1 \%$ & $35.1 \%$ & $95.4 \%$ & $97.4 \%$ \\
\hline & 4.0 & 0.018 & $(0.503)$ & 0.497 & $(0.755)$ & 1.714 & $(1.090)$ & 4.686 & $(1.297)$ \\
\hline & & -1.144 & $(0.564)$ & -0.598 & $(0.842)$ & 0.790 & (1.215) & 4.220 & (1.433) \\
\hline & & $1.2 \%$ & $2.2 \%$ & $7.3 \%$ & $13.1 \%$ & $48.2 \%$ & $60.9 \%$ & $99.1 \%$ & $99.8 \%$ \\
\hline & & $0.1 \%$ & $0.2 \%$ & $2.1 \%$ & $3.0 \%$ & $22.0 \%$ & $31.4 \%$ & $97.0 \%$ & $98.0 \%$ \\
\hline & F-test & $5.1 \%$ & $10.1 \%$ & $69.5 \%$ & $79.2 \%$ & $99.7 \%$ & $99.9 \%$ & $100.0 \%$ & $100.0 \%$ \\
\hline 200 & 0.25 & 0.007 & $(0.915)$ & 0.301 & $(0.951)$ & 1.080 & $(1.015)$ & 3.018 & $(0.970)$ \\
\hline & & -0.308 & $(0.964)$ & 0.010 & $(1.002)$ & 0.855 & (1.071) & 2.974 & $(1.016)$ \\
\hline & & $4.3 \%$ & $9.6 \%$ & $7.9 \%$ & $15.6 \%$ & $28.8 \%$ & $42.6 \%$ & $91.8 \%$ & $95.6 \%$ \\
\hline & & $2.4 \%$ & $5.6 \%$ & $5.7 \%$ & $11.1 \%$ & $23.7 \%$ & $34.9 \%$ & $90.3 \%$ & $94.8 \%$ \\
\hline & 0.5 & 0.012 & $\overline{(0.901)}$ & 0.425 & $(0.974)$ & 1.520 & $\overline{(1.109)}$ & 4.227 & $(1.134)$ \\
\hline & & -0.430 & $(0.951)$ & 0.017 & (1.029) & 1.205 & (1.174) & 4.164 & (1.189) \\
\hline & & $5.4 \%$ & $9.6 \%$ & $11.8 \%$ & $18.5 \%$ & $44.2 \%$ & $56.8 \%$ & $98.7 \%$ & $99.4 \%$ \\
\hline & & $2.5 \%$ & $5.5 \%$ & $7.1 \%$ & $12.3 \%$ & $34.5 \%$ & $45.8 \%$ & $98.3 \%$ & $99.1 \%$ \\
\hline & 1.0 & 0.015 & $(0.856)$ & 0.596 & $\overline{(1.011)}$ & 2.129 & $(1.271)$ & 5.898 & $(1.397)$ \\
\hline & & -0.604 & $(0.905)$ & 0.024 & $(1.072)$ & 1.685 & $(1.347)$ & 5.804 & (1.467) \\
\hline & & $4.5 \%$ & $8.2 \%$ & $15.0 \%$ & $23.7 \%$ & $62.4 \%$ & $72.6 \%$ & $99.8 \%$ & $99.9 \%$ \\
\hline & & $1.5 \%$ & $3.3 \%$ & $7.5 \%$ & $12.5 \%$ & $48.2 \%$ & $59.2 \%$ & $99.7 \%$ & $99.8 \%$ \\
\hline & 2.0 & 0.016 & $\overline{(0.763)}$ & 0.807 & $\overline{(1.057)}$ & 2.877 & $(1.481)$ & 7.944 & $(1.725)$ \\
\hline & & -0.848 & $(0.809)$ & 0.005 & (1.123) & 2.247 & (1.573) & 7.780 & (1.816) \\
\hline & & $2.9 \%$ & $6.6 \%$ & $19.2 \%$ & $27.8 \%$ & $79.5 \%$ & $86.3 \%$ & $100.0 \%$ & $100.0 \%$ \\
\hline & & $0.6 \%$ & $1.4 \%$ & $8.3 \%$ & $13.1 \%$ & $62.9 \%$ & $71.9 \%$ & $100.0 \%$ & $100.0 \%$ \\
\hline & 4.0 & 0.019 & $(0.558)$ & 0.949 & $(0.967)$ & 3.364 & $(1.496)$ & 9.245 & $(1.830)$ \\
\hline & & -1.153 & $(0.596)$ & -0.150 & (1.030) & 2.461 & (1.593) & 8.868 & (1.935) \\
\hline & & $0.9 \%$ & $2.5 \%$ & $20.8 \%$ & $31.0 \%$ & $87.5 \%$ & $93.1 \%$ & $100.0 \%$ & $100.0 \%$ \\
\hline & & $0.1 \%$ & $0.1 \%$ & $6.6 \%$ & $9.9 \%$ & $69.5 \%$ & $77.0 \%$ & $100.0 \%$ & $100.0 \%$ \\
\hline & F-test & $5.2 \%$ & $10.6 \%$ & $93.1 \%$ & $96.9 \%$ & $100.0 \%$ & $100.0 \%$ & $100.0 \%$ & $100.0 \%$ \\
\hline
\end{tabular}


Table 2: Sinus Alternatives

\begin{tabular}{|c|c|c|c|c|c|c|c|c|c|}
\hline$n$ & $c_{2}$ & \multicolumn{2}{|c|}{ DGP4 } & \multicolumn{2}{|c|}{ DGP5 } & \multicolumn{2}{|c|}{ DGP6 } & \multicolumn{2}{|c|}{ DGP7 } \\
\hline \multirow[t]{4}{*}{100} & \multirow[t]{4}{*}{0.25} & 0.563 & $(0.979)$ & 0.564 & $(0.982)$ & 0.571 & $(0.984)$ & 0.540 & $(0.971)$ \\
\hline & & 0.288 & (1.068) & 0.291 & $(1.072)$ & 0.297 & $(1.077)$ & 0.265 & $(1.065)$ \\
\hline & & $15.0 \%$ & $24.7 \%$ & $15.0 \%$ & $25.3 \%$ & $15.4 \%$ & $25.4 \%$ & $14.4 \%$ & $24.3 \%$ \\
\hline & & $11.2 \%$ & $19.9 \%$ & $11.7 \%$ & $20.2 \%$ & $12.4 \%$ & $20.4 \%$ & $11.0 \%$ & $19.1 \%$ \\
\hline & \multirow[t]{4}{*}{0.5} & 0.705 & $(1.007)$ & 0.798 & $(1.041)$ & 0.815 & $\overline{(1.053)}$ & 0.773 & $(1.039)$ \\
\hline & & 0.319 & $(1.100)$ & 0.422 & $(1.136)$ & 0.441 & (1.152) & 0.397 & (1.139) \\
\hline & & $18.2 \%$ & $28.5 \%$ & $22.0 \%$ & $31.7 \%$ & $22.0 \%$ & $32.6 \%$ & $20.6 \%$ & $31.2 \%$ \\
\hline & & $12.6 \%$ & $20.1 \%$ & $16.0 \%$ & $23.3 \%$ & $16.0 \%$ & $23.5 \%$ & $14.6 \%$ & $22.6 \%$ \\
\hline & \multirow[t]{4}{*}{1.0} & 0.598 & $(0.914)$ & 1.010 & $(1.086)$ & 1.093 & $(1.130)$ & 1.055 & $(1.116)$ \\
\hline & & 0.025 & $(1.000)$ & 0.478 & $(1.185)$ & 0.571 & $(1.234)$ & 0.532 & $(1.222)$ \\
\hline & & $13.2 \%$ & $22.2 \%$ & $26.6 \%$ & $37.5 \%$ & $29.9 \%$ & $40.8 \%$ & $28.6 \%$ & $38.8 \%$ \\
\hline & & $6.6 \%$ & $11.6 \%$ & $16.8 \%$ & $23.8 \%$ & $18.7 \%$ & $26.9 \%$ & $18.1 \%$ & $26.2 \%$ \\
\hline & \multirow[t]{4}{*}{2.0} & -0.030 & $(0.705)$ & 0.848 & $(0.944)$ & 1.232 & $(1.124)$ & 1.296 & $(1.155)$ \\
\hline & & -0.906 & $(0.779)$ & 0.056 & $(1.033)$ & 0.479 & (1.228) & 0.555 & $(1.265)$ \\
\hline & & $2.2 \%$ & $4.4 \%$ & $18.1 \%$ & $27.9 \%$ & $31.9 \%$ & $44.0 \%$ & $35.2 \%$ & $46.1 \%$ \\
\hline & & $0.6 \%$ & $1.0 \%$ & $7.5 \%$ & $12.5 \%$ & $16.5 \%$ & $23.0 \%$ & $17.8 \%$ & $26.0 \%$ \\
\hline & \multirow[t]{4}{*}{4.0} & 0.050 & $(0.497)$ & 0.046 & $(0.541)$ & 0.713 & $(0.791)$ & 1.071 & $(0.922)$ \\
\hline & & -1.107 & $(0.558)$ & -1.116 & $(0.602)$ & -0.384 & $(0.871)$ & 0.013 & (1.018) \\
\hline & & $0.7 \%$ & $2.3 \%$ & $1.3 \%$ & $2.8 \%$ & $11.8 \%$ & $20.6 \%$ & $23.4 \%$ & $36.3 \%$ \\
\hline & & $0.2 \%$ & $0.2 \%$ & $0.2 \%$ & $0.3 \%$ & $2.5 \%$ & $4.3 \%$ & $6.5 \%$ & $10.8 \%$ \\
\hline & F-test & $4.6 \%$ & $9.9 \%$ & $5.5 \%$ & $9.9 \%$ & $22.2 \%$ & $32.1 \%$ & $60.8 \%$ & $72.3 \%$ \\
\hline \multirow[t]{4}{*}{200} & \multirow[t]{4}{*}{0.25} & 1.068 & $(1.054)$ & 1.078 & $(1.058)$ & 1.073 & $(1.047)$ & 1.005 & $(1.033)$ \\
\hline & & 0.813 & (1.109) & 0.825 & (1.114) & 0.821 & $(1.103)$ & 0.750 & (1.089) \\
\hline & & $29.8 \%$ & $41.6 \%$ & $28.9 \%$ & $42.2 \%$ & $29.4 \%$ & $42.1 \%$ & $27.5 \%$ & $39.8 \%$ \\
\hline & & $23.6 \%$ & $33.6 \%$ & $23.1 \%$ & $33.9 \%$ & $22.9 \%$ & $33.9 \%$ & $21.3 \%$ & $31.2 \%$ \\
\hline & \multirow[t]{4}{*}{0.5} & 1.361 & $(1.148)$ & 1.482 & $(1.172)$ & 1.498 & $(1.165)$ & 1.408 & $(1.142)$ \\
\hline & & 0.996 & (1.209) & 1.126 & $(1.235)$ & 1.143 & $(1.229)$ & 1.051 & $(1.205)$ \\
\hline & & $38.6 \%$ & $50.5 \%$ & $42.6 \%$ & $54.5 \%$ & $43.6 \%$ & $55.0 \%$ & $40.2 \%$ & $52.6 \%$ \\
\hline & & $28.9 \%$ & $39.3 \%$ & $32.3 \%$ & $43.4 \%$ & $32.9 \%$ & $43.5 \%$ & $29.7 \%$ & $40.6 \%$ \\
\hline & \multirow[t]{4}{*}{1.0} & 1.305 & $\overline{(1.113)}$ & 1.911 & $(1.298)$ & 2.027 & $(1.339)$ & 1.937 & $(1.313)$ \\
\hline & & 0.759 & (1.172) & 1.401 & $(1.367)$ & 1.525 & (1.412) & 1.433 & $(1.386)$ \\
\hline & & $36.3 \%$ & $47.8 \%$ & $54.0 \%$ & $66.6 \%$ & $57.8 \%$ & $70.0 \%$ & $55.1 \%$ & $66.2 \%$ \\
\hline & & $21.5 \%$ & $31.6 \%$ & $39.5 \%$ & $49.7 \%$ & $43.4 \%$ & $53.2 \%$ & $41.0 \%$ & $50.4 \%$ \\
\hline & \multirow[t]{4}{*}{2.0} & 0.144 & $\overline{(0.798)}$ & 1.837 & $(1.238)$ & 2.418 & $(1.460)$ & 2.465 & $(1.482)$ \\
\hline & & -0.715 & $(0.847)$ & 1.076 & (1.304) & 1.691 & $(1.540)$ & 1.746 & $(1.566)$ \\
\hline & & $4.7 \%$ & $8.6 \%$ & $52.4 \%$ & $64.4 \%$ & $67.8 \%$ & $77.3 \%$ & $69.0 \%$ & $77.7 \%$ \\
\hline & & $1.0 \%$ & $2.1 \%$ & $30.1 \%$ & $40.8 \%$ & $47.1 \%$ & $56.5 \%$ & $48.2 \%$ & $58.3 \%$ \\
\hline & \multirow[t]{4}{*}{4.0} & 0.088 & $(0.571)$ & 0.362 & $(0.703)$ & 1.722 & $(1.136)$ & 2.299 & $(1.305)$ \\
\hline & & -1.078 & $(0.612)$ & -0.792 & $(0.745)$ & 0.647 & $(1.200)$ & 1.261 & (1.382) \\
\hline & & $1.5 \%$ & $3.2 \%$ & $5.9 \%$ & $10.6 \%$ & $47.4 \%$ & $61.4 \%$ & $67.1 \%$ & $76.8 \%$ \\
\hline & & $0.1 \%$ & $0.2 \%$ & $0.4 \%$ & $1.1 \%$ & $18.7 \%$ & $26.5 \%$ & $35.2 \%$ & $44.7 \%$ \\
\hline & F-test & $5.1 \%$ & $10.3 \%$ & $5.3 \%$ & $11.2 \%$ & $34.0 \%$ & $46.6 \%$ & $85.6 \%$ & $91.3 \%$ \\
\hline
\end{tabular}

